>> Title

Surveillance of in situ tumor arrays reveals early environmental control of cancer immunity

>>>Authors

5 Guadalupe Ortiz-Muñoz ${ }^{*}$, Markus Brown ${ }^{1 *}$, Catherine B. Carbone ${ }^{1 *}$, Joaquin Pechuan-Jorge ${ }^{1 *}$, Alex T.

6 Ritter $^{1}$, Darya Orlova ${ }^{1}$, Shreya R. Mantri ${ }^{1}$, Angela Yang ${ }^{4}$, Jonas Doerr ${ }^{2}$, Sanjeev Mariathasan ${ }^{3}$, Yulei

7 Wang $^{3}$, Shannon Turley ${ }^{1}$, Carlos Gomez-Roca ${ }^{6}$, Carlos Eduardo de Andrea ${ }^{7}$, David Spigel ${ }^{8}$, Thomas $\mathrm{Wu}^{5}$,

8 Zora Modrusan $^{4}$, Richard Price ${ }^{3}, \operatorname{Ira~Mellman}^{1}$, Christine Moussion $^{1} \dagger$

10 >>>Affiliations

$11{ }^{1}$ Department of Cancer Immunology, Genentech, Inc., South San Francisco, CA, USA

$12{ }^{2}$ Department of Molecular Biology, Genentech, Inc., South San Francisco, CA, USA

$13{ }^{3}$ Department of Oncology Biomarker Development, Genentech, Inc., South San Francisco, CA, USA

$14{ }^{4}$ Department of Microchemistry, Proteomics and Lipidomics, Genentech, Inc., South San Francisco, CA,

$15{ }^{5}$ Department of Bioinformatics and Computational Biology Genentech, Inc., South San Francisco, CA,

$16{ }^{6}$ IUCT, Institut Universitaire du Cancer de Toulouse, France

$17 \quad{ }^{7}$ Universidad de Navarra, Pamplona, Spain

$18{ }^{8}$ Sarah Cannon Research Institute, Nashville, USA

$19 *$ These authors contributed equally to this work

$20 †$ Email: moussion.christine@gene.com 
>>>Summary

The immune phenotype of a tumor is a key predictor of its response to immunotherapy ${ }^{1-4}$.

Patients who respond to immune checkpoint blockade generally present with tumors that are infiltrated by activated $\mathrm{T}$ cells, a tumor-immune phenotype referred to as 'immune inflamed ${ }^{5-7}$. patients presents with tumors that lack T cells ('immune desert') or that exclude $\mathrm{T}$ cells in the periphery of the tumor islet ('immune excluded') ${ }^{8}$. Despite the importance of these tumor-immune phenotypes in patients, little is known about their development, heterogeneity or dynamics due to an inability to model these features pre-clinically.

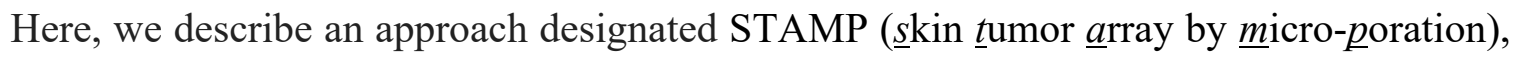
which combines in vivo noninvasive, high-throughput time-lapse imaging with excisional biopsies and next generation sequencing to characterize the establishment of the immunological niche and

38 follow its evolution during immunotherapy. STAMP involves the seeding of dozens to hundreds

39 clonal tumors in the superficial dermis of a single mouse ear that can be visualized in situ over weeks to months. Using this approach, we found that genetically identical tumors could display

41 surprisingly different immune phenotypes. Although individual tumors of the same array were

42 populated by the same $\mathrm{T}$ cell clonotypes, regression or progression of individual tumors were

43 associated with distinct patterns of spatial organization of the T cells. In situ imaging of $14 \mathrm{~K}$

44 tumors revealed that immune phenotypes were not static over-time but could rather evolve with

45 tumor growth and response to treatment. Therapy-induced or spontaneous early conversion to the 46 immune inflamed phenotype correlated with tumor regression and enhanced cytotoxic $\mathrm{T}$ cell

47 activity. Therefore, STAMP provides a flexible approach to study the relationship between tumor

48 evolution, immune cell dynamics, and tumor microenvironment with therapeutic response. 
>>>Main text

The STAMP technique uses an infrared laser (ER:YAG) ${ }^{9}$ to create an array of hundreds of

51 pores in the superficial dermis of a mouse ear pinna (Fig. 1a). Tumor cells from a clonal line

52 (Fig.1a, Extended-Data Fig.1a-c) expressing a fluorescent reporter are seeded into each pore and

53 subsequent tumor growth is monitored over the next 4-6 weeks using live imaging. STAMP can

54 successfully implant orthotopic (melanoma) (Extended-Data Fig.1d-f) or heterotopic (mammary,

55 pancreas, lung and colon carcinoma) tumor cell lines in the dermis of the mouse ear (Fig.1b) or

56 abdomen (Extended-Data Fig.2b). To automatically track large numbers of tumors over time, we

57 developed an image analysis pipeline based on machine learning (Extended-Data Fig.1g-m) that

58 quantifies 1) total tumor burden per animal over time, 2) growth of individual microtumors in the

59 array, and 3) tumor rejection rate.

60

\section{Local $\mathbf{T}$ cell-mediated rejection of clonal skin tumor array}

To characterize the immune infiltration into STAMP tumors we implanted $\mathrm{KPP}^{10}$ clonal

63 cell line (pancreatic ductal adenocarcinoma) subcutaneously or using ear STAMP(Fig.1c) and

64 found similar immune infiltrates regardless of implantation site. Immune cell profiling of ear

65 STAMP tumors was performed by flow cytometry analysis of ears with tumor-bearing vs tumor-

66 free micropores and demonstrated a significant recruitment of myeloid (macrophages, monocytes,

67 neutrophils, dendritic cells) and lymphoid populations (CD4, CD8 T cells) (Fig.1d). To assess the

68 role of adaptive immunity in controlling tumor growth, a STAMP-array of mouse KPP-EGFP

69 tumors was implanted into WT or immunodeficient Rag2-/- mice (Fig.1e). Though WT and Rag-

70 2-deficient animals displayed comparable initial tumor burdens (Fig.1f), after 14 days 
71 immunocompetent mice demonstrated a local rejection of $30 \%$ of individual tumors from the array,

72 while immunodeficient animals failed to reject tumors (Fig.1g-h). tumors in Rag-2-deficient mice and simultaneously reconstituted the mice with naïve tdTomato+ $\mathrm{T}$ cells from either WT mice, which contain a polyclonal population of T cells, or OT-I mice, which contain a monoclonal population of ovalbumin-specific CD8 T cells (Fig. 1i-k). In animals reconstituted with WT tdTomato+ T cells, T cells were recruited to the tumor sites as early as 6 rejection. However, in animals reconstituted with antigen-mismatched tdTomato $+\mathrm{T}$ cells from OT-I mice, T cells were not substantially recruited to tumor sites (Fig.1j-k) and tumors were not rejected (Fig.1i, Extended-Data Fig.1o, Extended-DataVideo1). As KPP-EGFP tumor cells do not express ovalbumin, we can conclude that bystander $\mathrm{T}$ cells were not sufficient to promote tumor rejection. Our findings support a role of antigen-specific T cells recruited into STAMP tumors in mediating spontaneous local rejection.

Heterogeneity and clinical relevance of mouse STAMP tumor-immune phenotypes

To further elucidate drivers of local tumor rejection, we characterized the spatial distribution of $\mathrm{T}$ cells in individual tumors. As indicated by fluorescence microscopy, a combination of immune inflamed, excluded and desert tumors were present within each array at

90 all analyzed time points (Fig.2a, Extended-Data Fig.2a-b, Extended-DataVideo2). The ability to

91 monitor individual tumors over time led to the definition of a new late onset phenotype, termed

92 "rejected tumor", in which EGFP+ tumor cell disappeared leaving a cluster of tdTomato+ T cells

93 for several weeks after tumor rejection. Importantly, by comparison, classical immunophenotyping 
94 of tumor biopsies by flow cytometry would not have allowed these diverse phenotypes to have

95 been distinguished (Fig.2c-d, Extended-Data-Fig.2c).

As the tumors from the array share the same clonal origin and are implanted simultaneously

97 at a single site in a single mouse, it was remarkable that distinct tumor immune phenotypes could

98 develop. We therefore next confirmed that heterogeneous $\mathrm{T}$ cell distribution in tumor arrays was

99 not an artifact of the STAMP method. For this purpose, we examined if different immune

100 phenotypes would also develop using a model dependent on spontaneous tumor seeding. The

101 immune phenotypes of lung metastatic tumors were monitored following intravenous seeding of

102 clonal KPP-EGFP tumor cells in Rag2-/- mice reconstituted with TdTomato+ T cells. As observed

103 in STAMP, the full spectrum of tumor-immune phenotypes was observed in lung nodules imaged

104 after optical clearing (Fig.2b, Extended-DataVideo3). Thus, our findings demonstrate clonally-

105 derived tumor cells have the unanticipated ability to develop heterogeneous immune phenotypes

106 independently of variations in either host or tumor genetics

107 To elucidate the basis for the diversity of immune phenotypes, we next explored the

108 biological pathways associated with each tumor niche. STAMP arrays were first imaged to classify

109 tumors by immune phenotype and individual tumors were then isolated by micro-punch biopsy for

110 bulk RNA sequencing (Fig.2c-e). Principal Component Analysis revealed that different immune

111 phenotypes constitute distinct transcriptional clusters (Fig.2e). Differential expression analysis of

112 genes (Extended-Data Fig2.d) and pathways (Fig.2f) identified gene signatures enriched in

113 immune inflamed, excluded, and desert tumors that were stable over time (Extended-Data Fig2.e).

114 The gene signatures enriched for each immune-phenotype of STAMP tumors were then compared

115 with pathways enriched for tumor-immune phenotypes in clinical samples using bulk RNAseq of

116 human ovarian (Fig.2g) and bladder FFPE tumors from clinical trials (ICON7 phase III and 
117 IMvigor210 phase II, respectively) (Extended-Data Fig.2e-f) that were classified as desert, 118 excluded, or inflamed based on CD8 and pan-cytokeratin staining.

119 Inflamed tumors from both mouse STAMP and human tumors were enriched for IFN $\alpha$,

120 IFN $\gamma$, and allograft rejection signatures and were also characterized by a downregulation of the

121 TGF- $\beta^{17}$ and NOTCH pathways. As previously described, T cell and interferon signatures are both

122 well validated drivers of the immune inflamed phenotype ${ }^{18}$ as is downregulation of TGF- $\beta$

123 pathway $^{11}$. Immune excluded tumors were enriched for epithelial-mesenchymal transition (EMT),

124 myogenesis, angiogenesis, hypoxia and UV response signatures. EMT, myogenesis and 125 angiogenesis enrichment in excluded tumors is consistent with the enrichment in stromal and 126 myofibroblast components described previously ${ }^{12,13}$. Finally, immune desert tumors were enriched 127 for $\mathrm{WNT} / \beta$-catenin ${ }^{22}$ and Hedgehog pathways ${ }^{23}$ and showed a downregulation of immune 128 signatures including allograft rejection, $\mathrm{T}$ cell, and interferons. WNT/ $\beta$-catenin has previously 129 been associated with poor immune cell infiltration ${ }^{14,15}$. The overlap of significant pathways 130 enriched in mouse STAMP tumors and human tumors of the same immune phenotypes held across 131 different human cancer types (ovarian, bladder and lung) (Extended-Data Fig.2e-f and data not 132 shown) Thus, the similarity of gene signatures associated with the three immune phenotypes in 133 both human and STAMP tumors suggests that STAMP may recapitulate the general mechanisms 134 underlying this aspect of tumor diversity observed clinically.

\section{Immune phenotype determines $\mathbf{T}$ cell function regardless of $\mathbf{T}$ cell clonotype}

Given the heterogeneity in spatial organization of $\mathrm{T}$ cell infiltrates among individual 138 tumors, we next asked if these variations correlated with qualitative differences in local $\mathrm{T}$ cell 139 responses. Although each tumor was derived from a common cell line and the T cells likely 
140 derived from the same draining lymph nodes, it was possible that differences in T cell infiltration

141 and tumor growth control reflected differences in local T cell receptor clonotype profiles. To this

142 end, we performed 5'single cell RNAseq and TCR-seq of tumor punch biopsies harvested from

143 the same mouse 8 days after tumor implantation; individual tumors of the same immune phenotype

144 were pooled to provide sufficient numbers of cells for the analysis.

145 Single cell analysis identified twelve clusters of T cells which included four CD8 T cell

146 clusters: two CD8 T effector cell clusters, one CD8 $\mathrm{T}_{\mathrm{RM}}$ cell cluster and one mitotic CD8 T cell

147 cluster. The remaining eight sub-clusters were CD4 T cell clusters, including two mitotic CD4 T

148 cell clusters, one Treg cluster and five CD4 effector T cells clusters (Fig.3a,b; Extended-Data

149 Fig.3a-d). Interestingly, we did not observe any difference in absolute or relative abundance of T

150 cell subsets across tumors with inflamed, excluded and resolved phenotypes (Fig.3c,d; Extended-

151 Data Fig.3e,f). Desert tumors, as expected, were devoid of most T cells populations.

152 The relative and total abundance of $\mathrm{T}$ cell clonotypes was also shared across inflamed,

153 excluded and rejected phenotypes (Extended-Data Fig.3g-j). We identified seven

154 immunodominant clonotypes each of them present at comparable frequencies among inflamed,

155 excluded, and rejected tumors (Fig.3d,e; Extended-Data Fig.3h-i). However, we found that

156 identical T cell clonotypes exhibit divergent gene expression profiles when present in tumors with

157 differing immune phenotype. Differential gene and pathway analysis of immunodominant $\mathrm{T}$ cell

158 clonotypes showed that $\mathrm{T}$ cells clones present in inflamed tumors were characterized by signatures

159 indicating an increased translation and mitochondrial biogenesis compared to the same $\mathrm{T}$ cells

160 clones located in excluded tumors. (Fig.3f; Extended-Data Fig3k,n).

161 Because mitochondrial dynamics control $\mathrm{T}$ cell fate ${ }^{16-18}$ and translational activity correlates

162 with the activation and differentiation state of the effector $\mathrm{T}$ cells ${ }^{19}$, we investigated the 
163 functionality of the $\mathrm{T}$ cells present in immune inflamed and immune excluded tumors. We

164 developed a clonal KPP tumor cell line expressing the calcium sensor ${ }^{20}$ GCaMP6 that emits green

165 fluorescence when the intracellular $\mathrm{Ca}^{2+}$ concentration rises, as occurs upon effector $\mathrm{T}$ cell attack

166 and membrane perforation. Repetitive $\mathrm{T}$ cell attacks highlighted by repetitive $\mathrm{Ca}^{2+}$ flashes in tumor

167 cells were followed up by tumor cell death highlighted by Propidium Iodide uptake (Fig.3g-i;

168 Extended-Data Fig.3o-s). Therefore, the occurrence of green flashes in tumor cells correlate with

169 T cell cytolytic activity. We validated the sensitivity and the specificity of calcium flashes in

170 response to $\mathrm{T}$ cell killing in vitro using KPP organoids (Fig.3h,i; Extended-Data Fig.3p-s;

171 Extended-DataVideos4,5) and in vivo using STAMP microtumors (Extended-Data Fig.3t,u;

172 Extended-DataVideos6-8). We then quantified the difference in $\mathrm{Ca}^{2+}$ flashes between immune

173 inflamed and immune excluded tumors from STAMP arrays at 8 days after implantation and

174 further correlated it with their tumor growth rates (Fig.3j-n; Extended-DataVideos9-10). The

175 flashing index of inflamed tumors was found to be significantly higher as compared to excluded 176 tumors (Fig.3m) despite comparable total T cell abundances (Fig.31). Indeed, a high flashing index

177 corresponded to an immune inflamed phenotype and a slow tumor growth rate (Fig. 3n; Extended-

178 Data Fig.3v,w).

179 Taken together, these results indicate that $\mathrm{T}$ cells of the same TCR clonotype exhibit

180 different functional capacity when localized to immune inflamed vs immune excluded tumors,

181 emphasizing a determinative role for the tumor microenvironment in shaping the activity and fate

182 of endogenous effector T cells. 
Finally, we explored how the spatial distribution of $\mathrm{T}$ cells predict tumor progression or rejection during Immunotherapy. Although human and mouse tumors are well known to adopt one

187 or another immune phenotype, it remains unclear whether these states are stable or dynamic.

188 STAMP provides an opportunity to determine how immune phenotypes evolve with 189 immunomodulatory therapy. As immune excluded and immune desert STAMP tumors exhibited 190 an upregulation of the TGFb pathway (Fig.2g), we hypothesized that TGFb could restricts T cell 191 entry into the tumor parenchyma. It has been previously shown that both $\mathrm{T}$ cell recruitment and 192 therapeutic benefit can be elicited in mice by treatment with $\mathrm{TGFb}$ antagonists in combination 193 with checkpoint blockade anti-PD-L1 ${ }^{21}{ }^{11}$. We treated STAMP tumor-bearing mice with anti-PD-

194 L1, anti-TGF- $\beta$ or a combination of both. While treatment with anti-PD-L1 or anti-TGF- $\beta$ alone 195 failed to improve tumor rejection rates, combination therapy led to a $73.3 \%$ overall response rate 196 (ORR) in immunocompetent mice (Extended-Data Fig.4a-c) and 69\% O.R.R in RAG2-/- mice 197 reconstituted with adoptively transferred T cells (Fig.4a-b, Extended-Data Fig.4d).

199 spontaneously in the control arm (Fig.4b-c), we next quantified T cell abundance in tumors as a 200 function of time. We performed daily imaging of STAMP KPP-EGFP tumors implanted in 201 RAG2-/- mice reconstituted with tdTomato+ T cells and treated with anti-TGF $\beta$ and anti-PD-L1. 202 As shown in Fig. 4d, tumors in combination-treated mice exhibited a significant increase in total 203 T cells starting at Day 10. Although STAMP tumors in mice treated with anti-PD-L1 alone also 204 exhibited increases in CD8 T cell infiltration (Extended-Data Fig.4e), only the combination 205 therapy led to tumor rejection (Fig.4a).

206 Having shown that a simple increase in total T cells at the tumor site was insufficient to 207 induce efficient tumor rejection, we next sought to identify other immunological features that 
208 precede efficient rejection. We therefore examined the spatial distribution of $\mathrm{T}$ cells over time in

209 response to the combination therapy using a high content image analysis pipeline to automatically

210 classify tumors as immune inflamed, excluded or desert. T cell phenotype classifications could

211 then be overlaid with tumor growth curves to create a trajectory representation of tumor fate

212 (Extended-Data Fig.4f-h). Tumor trajectories were plotted using hierarchically clustered heatmaps

213 illustrating tumor growth rate (Fig.4e), median T cell abundance (Fig.4f), and tumor immune

214 phenotype over time (Fig.4g). The analysis of $1.2 \mathrm{~K}$ individual tumors implanted in 20 animals

215 showed that responding tumors (C.R.+P.R.) in both combination therapy-induced rejections

216 (turquoise) and spontaneous rejections (dark blue) shared similar trajectories over time, both

217 showing a negative growth rate and higher degree of T cell recruitment prior to tumor rejection.

218 To further characterize dynamic changes between immune phenotypes, we developed a

219 Markov chain model to compute changes in transition probability among the different states:

220 desert, excluded, inflamed, rejected tumor and animal euthanasia (Fig.4h, Extended-Data Fig.4i-

$221 \mathrm{k}$, Extended-DataTable1). We observed that responding tumors (CR+PR) were in general more

222 likely to transition from desert to inflamed and from excluded to inflamed than non-responding

223 tumors (SD+PD) regardless of the treatment (Extended-Data Fig.4i,j). The combination treatment

224 relative to control increased the probability of transition from excluded to inflamed and decreased

225 the probability of reverse transition from inflamed to excluded (Fig.4h). Additionally, the

226 probability of an inflamed or an excluded tumor becoming resolved was increased relative to

227 control if treated with combination immunotherapy (Fig.4h). These results suggest that differences

228 in tumor outcome may be associated with an increased probability of a tumor becoming inflamed,

229 and that such transitions may be promoted by combination immunotherapy 
To better understand which portion of the tumor trajectory determines tumor outcome, we

231 used a radial analysis to calculate an exclusion score (maximum $\mathrm{T}$ cell abundance in the tumor

232 core/maximum T cell abundance at the tumor periphery) for tumors over time (Fig.4i-k, Extended-

233 Data Fig.41). Although tumors increased their inflammatory features over time, the first $20 \%$ of

234 the trajectory of non-responding tumors ( $\mathrm{SD}+\mathrm{PD}$; red, magenta) became substantially more

235 excluded than responding tumors (CR+ PR; turquoise, blue) (Fig 4.k). Thus, if an individual tumor

236 became more excluded early in its trajectory, that tumor would be less likely to be rejected. This

237 analysis highlights the importance of early $\mathrm{T}$ cell infiltration into the tumor core, as a transition to

238 an inflamed phenotype late in a tumor's lifetime is less efficient at inducing tumor regression

239 (Fig.4k).

240 Finally, to identify deeper patterns in the dataset, we performed multivariate clustering 241 analysis using UMAP (Extended-Data Fig.4m-o) and SPADE tree (Fig.41-o, Extended-Data Fig4r-

$242 \mathrm{v}$ ) of $11 \mathrm{~K}$ images of individual tumors acquired between Day 6-17 (Extended-DataTable2) to

243 observe treatment-specific immunomodulation over time (Extended-Data Fig.4n,o) and to further

244 quantify it using the Earth Moving Distance analysis (dissimilarity score) for each treatment group

245 (Extended-Data Fig.4o-q). This multivariate analysis revealed that T cell dynamics of the anti-PD-

$246 \mathrm{~L} 1 /$ anti-TGFb combo treatment group has significantly smaller dissimilarity to the Immune

247 dynamics of responder tumors than any of the other treatment groups over time. Additionally,

248 SPADE trees generated a hierarchical clustering that revealed patterns of heterogeneity within the

249 treatment groups (Fig.41-o, Extended-Data Fig.4r,v) previously ignored by a simple phenotype

250 classification (inflamed, excluded, desert). As shown in Fig.41 the responders group splits into two

251 subpopulations of inflamed tumors (arrows): type I, which has a higher exclusion ratio and total T 
252 cell abundance, and type II, which had lower values (Fig.4m-o). Both subtypes had high 253 probability of response to therapy.

As summarized in Fig.4p, STAMP reveals a spatial and temporal control of Immune-

255 mediated tumor rejection relying on 1) increased total $\mathrm{T}$ cell abundance at the tumor site, 2)

256 transition to an inflamed phenotype, 3) earliest T cell infiltration in the tumor core. STAMP could

257 also be used to identify heterogenous patterns of response to therapeutics facilitating future 258 exploration of novel classes of tumors.

Evolution of tumor immune phenotype in human cancers

The analysis of STAMP tumors indicated that tumor immune phenotypes were capable of

262 dynamic evolution that determine their response to therapy. To assess whether a similar situation

263 might exist for human tumors, we analyzed on treatment biopsies taken from six patients with

264 various solid tumor indications both before and during treatment with checkpoint inhibitors

265 (Extended-DataTable3). The distribution of CD8 $\mathrm{T}$ cells was determined by

266 immunohistochemistry relative to the tumor nest. As shown in Fig.4q, it is apparent that the

267 immune phenotype of a tumor can convert over time with therapeutic intervention, and not always

268 from less to more inflamed. In the paired samples shown, examples of transitions immune desert

269 to excluded (NSCLC), desert to inflamed (maxillofacial), excluded to desert (melanoma I) and

270 excluded to excluded (melanoma II) can be seen. Understanding the spontaneous or CIT-induced

271 evolution of tumor Immune phenotypes in the clinic will be an important consideration in

272 predicting the likelihood of clinical response. Further modeling of tumor-immune dynamics by

273 STAMP and integration of multiple spatial and temporal variables ${ }^{22}$ may provide insight into the 
274 mechanisms determining tumor fate and provide the biomarkers that may guide therapy in human

275 cancer patients.

276

$277>>$ Methods

\section{STAMP (ㅁkin tumor $\underline{\operatorname{array}}$ by $\underline{m}$ icroporation) implantation}

279 Experimental procedures were performed in 8-12-week-old male mice under anesthesia 280 (60-100 mg/kg ketamine \& xylazine 5-10 mg/kg, intraperitoneal injection). Prior to microporation, 281 the ear hair was removed with Veet ${ }^{\circledR}$ depilatory cream then rinsed with water, dried, and the dorsal 282 side of the ear was immobilized with double-sided tape to expose the ventral side. Microporation 283 at $71 \mu \mathrm{m}$ depth and $9 \%$ pore density is performed by applying the P.L.E.A.S.E laser device (Pantec 284 Biosolutions, Liechtenstein) to the ventral side of the ear using a custom program previously 285 validated for the cell line.

286 After the microporation process, $150 \mu \mathrm{L}$ of a tumor cell suspension (EMT6-EGFP, CT26287 RFP, 4T1-mTagBFP2, KPP-EGFP, KPP-mTagBFP2, NSCLC-EGFP or B16F10) at 40-80x106 288 cells/mL diluted in phosphate buffered saline was applied to the ear covering the ventral side. Cells 289 were incubated for $30 \mathrm{~min}$ then the excess cell suspension was removed, pores were covered with 290 Matrigel (Corning, NY, USA) and incubated for 15 min until matrigel polymerized. Microtumor 291 growth becomes evident 5 to 12 days post tumor implantation, depending on the cell line.

293 Mice

The Genentech institutional animal care and use committee responsible for ethical 295 compliance approved all animal protocols. Mice were housed under specific pathogen-free 
conditions at Genentech animal facility, South San Francisco, CA. Animals between 8-12 weeks old that appeared healthy and free of obvious abnormalities were used for the study. 003291), and C57BL/6J (Stock No. 000664) animals were purchased from the Jackson were purchased from Taconic Biosciences (CT, USA).

CD4.cre.tg Rosa26.LSL.tdTomato.cki OT-I.TCR.tg (OT1-/- and OT1+/+) animals and E8I.CD8A.IRES.GFP.Cre.tg Rosa26.LSL.tdTomato.cki animals were bred in house.

Subcutaneous tumors.

For subcutaneous tumor inoculation, mice were injected subcutaneously with $0.5 \times 10^{6}$ KPP-EGFP cells in $100 \mu \mathrm{L}$ of a 1:1 dilution of phosphate buffered saline and Matrigel (Corning, NY, USA).

\section{CIT treatment.}

Animals were implanted with STAMP microtumors as described previously. Mice were

313 distributed into treatment groups to exclude cage effects and when possible, account for

314 differential initial tumor growth. Treatment was initiated one day post tumor implantation for 315 immunodeficient mice or 7 days post tumor implantation for immunocompetent mice. Animals 316 were treated every other day with isotype control antibodies (anti-gp120, mouse IgG clone 3E5, $31710 \mathrm{mg} / \mathrm{kg}$ ), anti-PD-L1 (mouse IgG1 clone 6E11, $10 \mathrm{mg} / \mathrm{kg}$ first dose followed by $5 \mathrm{mg} / \mathrm{kg}$ 318 thereafter), anti-TGF- $\beta$ (mouse IgG1 clone 1D11, $10 \mathrm{mg} / \mathrm{kg}$ ), or a combination of anti-PD-L1 
319 with anti-TGF- $\beta$. 5 days after treatment initiation, mice were imaged daily on a M205FA

320 stereoscope with a 1.0x PlanApo lens (10450028; Leica Microsystems, Germany) and ORCAII

321 Digital CCD (Hamamatsu Photonics, Japan) to monitor tumor growth and T cell progression. For

322 selected experiments, immunodeficient mice were injected intravenously with $4 \times 10^{6}$ isolated $\mathrm{T}$

323 cells-Tomato from CD4.cre.tg_Rosa26.LSL.tdTomato.cki_OT-I.TCR.tg as described below.

\section{Metastasis mouse model and imaging of whole mount tissue}

326 RAG-2-deficient mice were injected intravenously with $0.1 \times 10^{6}$ KPP-EGFP cells and $4 \times 10^{6}$

327 tdTomato + CD3 + T cells isolated as described below. 8 days post intravenous injection, mice were

328 euthanized, and $20 \mathrm{~mL}$ of cold $\mathrm{PBS} /$ heparin $5 \mathrm{U} / \mathrm{ml}$ solution was perfused directly into the right

329 ventricle using a 27-gauge needle. Lungs were isolated by dissection and tissues were fixed using

$3304 \%$ paraformaldehyde in phosphate buffered saline ${ }^{23}$. Tissue clearing was performed using the

331 FluoClearBABB approach ${ }^{24}$ and whole mount images were then acquired using a SP8 microscope

332 equipped with a white light laser and a HCX APO L 20x/0.95 IMM lens (Leica Microsystems,

333 Germany). Imaging data was analyzed on a workstation (Thinkmate, MA, USA) using Imaris

334 software (Bitplane, United Kingdom).

335 T cell isolation.

$336 \mathrm{~T}$ cells were isolated from C57BL/6J or CD4.cre.tg Rosa26.LSL.tdTomato.cki OT-

337 I.TCR.tg (OT1-/- and OT1+/+) mice. Spleens were collected and dissociated with the end of a 338 plunger from a $1 \mathrm{~mL}$ syringe into $10 \mathrm{~mL}$ of PBS before filtration through a $70 \mu \mathrm{m}$ cell strainer. T 339 cells were isolated for intravenous injection using an EasySep ${ }^{\mathrm{TM}}$ Mouse $\mathrm{T}$ Cell Isolation Kit 340 (Stemcell Technologies, Canada). $4 \times 10^{6}$ isolated $\mathrm{T}$ cells were injected intravenously per mouse. 
To generate CTLs from OT-I mice, splenocytes were isolated and stimulated with $10 \mathrm{nM}$

OVA257-264 peptide (AnaSpec, CA, USA) in complete media containing Gibco RPMI 1640

343 (Thermo Fisher Scientific, MA, USA) with 10\% Gibco fetal calf serum (Thermo Fisher Scientific,

344 MA, USA), $2 \mathrm{mM}$ L-glutamine, $50 \mathrm{U} / \mathrm{mL}$ Gibco penicillin/streptomycin (Thermo Fisher

345 Scientific, MA, USA), and $50 \mu \mathrm{M} \beta$-mercaptoethanol. Following 3 days of stimulation, cells were

346 resuspended in complete media with $10 \mathrm{IU} / \mathrm{mL}$ recombinant human IL-2 (rHIL-2). CTLs were

347 kept at a density of $0.5 \times 10^{6}$ cells $/ \mathrm{mL}$ and fresh complete media with rHIL-2 was added every 48

348 hours. CTLs were used between 6 and 8 days after primary in vitro stimulation.

\section{Flow cytometry}

Ear tissue was isolated using a $1 \mathrm{~mm}$ Miltex sterile disposable biopsy punch with plunger

352 (Integra Biosciences, NH, USA) from animals bearing STAMP microtumors or mock-implanted

353 control mice at either 8 or 18 days post tumor implantation. Tumors were digested in $500 \mu \mathrm{L}$

354 (STAMP microtumors) or $3000 \mathrm{~mL}$ (subcutaneous) of phosphate buffered saline containing 0.1

$355 \mathrm{mg} / \mathrm{mL}$ DNAse I (Roche, Switzerland) and collagenase D at $1 \mathrm{mg} / \mathrm{mL}$ (Roche, Switzerland) for 30

$356 \min$ at $37^{\circ} \mathrm{C}$ to obtain a single cell suspension.

$357 \quad$ For surface staining, cells from digested tumors were incubated with Fc block $(5 \mu \mathrm{g} / \mathrm{mL}$;

358 BD Biosciences, CA, USA; clone 2.4G2) and stained with antibody mix for 30 min and Viability

359 Dye eFluor 780 (eBioscience, CA, USA).

360 Antibodies were used at 1:200 dilution. Anti-mouse CD19 (clone B4), anti-mouse I-A/I-E

361 (clone M5/114.15.2), anti-mouse F4/80 (clone BM8), anti-mouse/human CD11b (clone M1/70),

362 anti-mouse Ly-6G (clone 1A8), anti-mouse Ly-6C (clone HK1.4), anti-mouse CD69 (clone

363 H1.2F3), anti-mouse CD25 (clone PC61), anti-mouse CD4 (clone RM4-5), anti-Mouse CD62L 
364 (clone MEL-14), and anti-mouse/human CD44 (clone IM7) antibodies were purchased from

365 Biolegend (CA, USA). CD45 anti-mouse (clone 30:F11), anti-mouse CD86 (clone GL1), CD11c 366 anti-mouse (clone N418), and anti-mouse CD8a (clone 53-6.7) antibodies were purchased from

367 ThermoFisher Scientific (MA, USA). CD3 anti-mouse (clone 17A2) antibody was purchased from 368 BD Biosciences (CA, USA). Live singlets cells subsets CD45+ were gated as follow: MHC class II + CD11c + F4/80- as DC or CD103+ or CD86+ activated DC; CD11c- CD11b+ F4/80+ as macrophages, CD11b+

371 Ly6G+ Ly6C ${ }^{\text {int }}$ as neutrophils; CD11b + Ly6G $^{\text {low }}$ Ly6C + as monocytes; CD3+ T cells were divided as CD3 + CD4 $+\mathrm{T}$ cells, CD3 $+\mathrm{CD} 8+\mathrm{T}$ cells, CD3 $+\mathrm{CD} 69+$ activated/resident $\mathrm{T}$ cells, CD3+ CD44+ CD62L- effector/effector memory T cells or CD3+ CD44- CD62L+ naive T cells.

Flow cytometry data were collected with a BD LSRFortessa cell analyzer (BD Biosciences, CA, USA) and analyzed using FlowJo Software (Version 10.2; FlowJo LLC, OR, USA).

\section{Whole Exome Sequencing Analyses}

We conducted whole exome sequencing of DNA isolated from the KPP-EGFP cell line harvested at the end of a culture passage, under the conditions for the experiments of this work, in order to ascertain the absence of major sub-clones explaining the diversity of immune phenotypes observed. A spleen sample from the mice strain used in our experiment were used as matched normal. Exome capture libraries were sequenced on HiSeq 2500 (Illumina) to generate $2 \times 75 \mathrm{bp}$

383 paired-end data, from the sequenced reads, variants were called using the following workflow.

384 Sequencing reads were mapped to UCSC mouse genome (GRCm38) using BWA software ${ }^{25}$ set to 385 default parameters. Local realignment, duplicate marking, and raw variant calling were performed 386 according to GATK best practices ${ }^{26}$. Somatic variant calling on tumor and its matched normal 
387 BAM file was performed using Strelka ${ }^{27}$. The resulting VAFs allowed us to construct histograms

388 to demonstrate clonality. Additionally, the $\mathrm{R}$ package neutralitytestr (https:

389 //github.com/marcjwilliams1/neutralitytestr) was used to show that low frequency variants

390 detected are indeed part of the neutral drift that any cultivation process entails.

STAMP microtumor sample preparation for bulk RNAseq of tumor biopsies

FOXN1-deficient nude mice with adoptively transferred tdTomato + CD3 + T cells bearing

395 Miltex sterile disposable biopsy punch with plunger (Integra Biosciences, NH, USA). Each tissue

396 biopsy was transferred to a separate $1.5 \mathrm{~mL}$ tube (Eppendorf, Germany) containing $0.25 \mathrm{~mL}$

397 Invitrogen TRIzol Reagent (Thermo Fisher Scientific, MA, USA). Biopsies were incubated in

398 TRIzol for 5 min with intermittent vortexing. $50 \mu \mathrm{L}$ chloroform (MilliporeSigma, MA, USA) was

399 added to the homogenate, vortexed for $20 \mathrm{sec}$, and incubated at $20-25^{\circ} \mathrm{C}$ for $2-3 \mathrm{~min}$. To accelerate

400 phase separation, samples were centrifuged at $10,000 \mathrm{xg}$ for $18 \mathrm{~min}$ at $4^{\circ} \mathrm{C}$. The aqueous (top)

401 phase was removed by aspiration and transferred to a clean $1.5 \mathrm{~mL}$ tube (Eppendorf, Germany). A

402 volume of $100 \%$ RNAse-free ethanol (MilliporeSigma, MA, USA) equal to the volume of the

403 aqueous layer was added, and the RNA was further isolated using a RNeasy Micro Kit (Qiagen,

404 Germany). Alternatively, an individual tumor biopsy was immersed in RNA later and the RNA

405 further extracted using a RNeasy Mini Kit (Qiagen, Germany).

407 STAMP microtumor bulk RNAseq analysis

408 RNA-sequencing data were analyzed using HTSeqGenie pipeline in BioConductor ${ }^{28}$ as follows:

409 first, reads with low nucleotide qualities (70\% of bases with quality $i 23)$ or matches to rRNA and 410 adapter sequences were removed. The remaining reads were aligned to the mouse reference 
411 genome GRCm38.p5 using GSNAP (PMID:20147302, 27008021) version '2013-10-10-v2',

412 allowing maximum of two mismatches per 75 base sequence (parameters: '-M 2 -n 10 -B 2 -i 1 -

413 N 1 -w 200000 -E 1 -pairmax-rna=200000 -clip-overlap'). Transcript annotation was based on

414 the Gencode genes database ${ }^{29}$. To quantify gene expression levels, the number of reads mapping

415 unambiguously to the exons of each gene was calculated. The resulting count matrix was filtered

416 for lowly expressed genes keeping the genes with at least 0.2 counts per million in more than six

417 samples. Principal component analysis was performed with the factoextra R package. Differential

418 gene expression analysis was performed using the voom and limma $\mathrm{R}$ packages ${ }^{30}$. To detect

419 signature differences amongst immune phenotypes, our contrasts compared the immune phenotype

420 at hand with the average of the other two. Volcano plots were constructed using the

421 EnhancedVolcano R package (https://github.com/kevinblighe/EnhancedVolcano). Gene set

422 enrichment analysis was performed on the log Fold Changes with using the GSEA function with

423 ClusterProfiler R package the Hallmark Gene Set Collection ${ }^{31}$. Pathways were considered 424 significant if their multiple testing corrected p-value was less that 0.2 . The same analysis was 425 performed on the clinical trial RNA seq data. Heatmaps of the normalized enrichment score were 426 constructed for those significant pathways was constructed using the ComplexHeatmap R 427 package $^{32}$.

\section{Sample preparation of sorted STAMP microtumors for scRNAseq}

430 FOXN1-deficient nude mice with adoptively transferred tdTomato $+\mathrm{CD} 3+\mathrm{T}$ cells bearing

431 KPP-EGFP STAMP microtumors were biopsied 8 days post tumor implantation using a $1 \mathrm{~mm}$

432 Miltex sterile disposable biopsy punch with plunger (Integra Biosciences, NH, USA). 3-6 pooled 433 tissue biopsies were moved into a precooled $1.5 \mathrm{~mL}$ tube (Eppendorf, Germany) containing $300 \mu \mathrm{L}$ 
434 digestion cocktail consisting of Gibco RPMI 1640 (Thermo Fisher Scientific, MA, USA), 0.1\%

435 Gibco fetal calf serum (Thermo Fisher Scientific, MA, USA), 0.1mg/ml Liberase TM (Roche,

436 Switzerland), 0.1mg/ml DNAse I (Roche, Switzerland), 32uM Gibco Actinomycin D (Thermo

437 Fisher Scientific, MA, USA). Tissues were incubated for $30 \mathrm{~min}$ at $37^{\circ} \mathrm{C}$ and $950 \mathrm{x}$ RPM on a

438 Thermoblock (Eppendorf, Germany) and mechanically dissociated every 10 min with a pipette.

439 To quench the digestion, the cell suspension was filtered through a $40 \mu \mathrm{m}$ mesh into pre-cooled

440 FACS filter tube containing quenching buffer of Gibco fetal calf serum (Thermo Fisher Scientific,

441 MA, USA) with 32 uM Gibco Actinomycin D (Thermo Fisher Scientific, MA, USA). The cell

442 suspension was centrifuged at 350xg for 8 min and resuspended in $400 \mu \mathrm{L}$ Gibco RPMI 1640

443 (Thermo Fisher Scientific, MA, USA) with $5 \mu \mathrm{M}$ CalceinBlue (Invitrogen, CA, USA) and a 1:200

444 dilution of Molecular Probes Fixable Live/Dead Near-IR Dead Cell Stain Kit (Thermo Fisher

445 Scientific, MA, USA). FACS was performed to isolate Calcein Blue-positive and L/D NearIR-

446 negative cells into a precooled $1.5 \mathrm{ml}$ Eppendorf tube containing $750 \mu \mathrm{L}$ collection buffer

447 consisting of Gibco RPMI 1640 (Thermo Fisher Scientific, MA, USA), 10\% Gibco fetal calf serum

448 (Thermo Fisher Scientific, MA, USA), 32 uM Gibco Actinomycin D (Thermo Fisher Scientific,

449 MA, USA). The cell number and viability were determined on a Vi-Cell XR cell viability analyzer

450 (Beckman Coulter, CA, USA) and scRNAseq library preparation was performed using dual single

451 cell mouse kit 5'/TCR according to the manufacturer's instructions (10x Genomics, CA, USA).

\section{3 scRNAseq analysis of STAMP microtumors}

454 Two independent experiments of STAMP microtumors were single cell sequenced using the 10x 455 platform (10x Genomics, CA, USA). The first experiment consisted of four microtumors of each 456 of the following phenotypes: immune infiltrated, immune excluded and immune desert isolated 
457 from the same animal to have a shared $\mathrm{T}$ cell repertoire across the different tumors. For the second 458 experiment, in addition to repeating the same sampling, we also sequenced tumors derived from a 459 mouse with no adoptively transferred CD3 + T cells and a mouse with only pores (no implantation 460 of tumor cells). For both experiments, paired TCR sequencing was performed. Single-cell RNA461 seq data was processed with cellranger count (CellRanger 3.0.2 from 10x Genomics) using a 462 custom reference package based on mouse reference genome GRCm38 and GENCODE ${ }^{29}$ gene $^{2}$ 463 models. Data analysis was done in R 3.6.2 and the Seurat package version 3.1.5 ${ }^{33}$. Only high 464 quality cells were retained for the posterior analysis, more concretely, we kept the cells with more 465 than 300 hundred genes detected, more than 1000 unique molecule identifiers (UMIs) and less 466 than $10 \%$ mitochondrial reads. This filtering step removed most of the doublets and empty cells. 467 From our single cell data, a preliminary clustering was performed using the standard Seurat SCT 468 transform workflow to determine the major cell populations in our samples. The tdTomato 469 positive clusters were analyzed using the standard SCT transform workflow from Seurat. Using 470 the talus plot $^{34}$ we determined that 60 principal components were enough to capture the signal in 471 data. With these retained components, we computed a UMAP embedding and the neighbors for 472 the clustering. Several clustering resolutions were calculated and a directed tree was constructed 473 reflecting the hierarchical relationships of the new clusters upon increasing the resolution ${ }^{35}$. A 474 resolution of 1.5 was considered optimal and the main clusters were identified by means of 475 expression markers of known biology. Markers for each cluster were identified using the 476 Seurat::FindAllMarkers() method with default parameters, comparing all cells in a particular 477 cluster to the rest of cells in the dataset and accessing significantly differential gene expression 478 using Wilcoxon's rank sum test and FDR correction for multiple testing. We used Seurat's plotting 479 functionlalities for most plots. Maker heatmaps were generated with the ComplexHeatmap R 
480 package ${ }^{32}$ using results from the AverageExpression function of Seurat as input (scaled to relative

481 expression per gene: z-scored per row). Clonotype analyses and integration with Seurat were done

482 using the scRepertoire R package ${ }^{36}$. For the fine structure of the clusters, we were unable to identify

483 a parallel with known T cell subtypes and therefore the clusters were named by their top positive

484 markers. We performed differential gene expression using Seurat::FindMarkers() on each T cell

485 cluster comparing the inflamed to the excluded immune phenotypes using batch as a latent variable

486 and the negative binomial test. We reported the significant genes log Fold Changes values as a z-

487 score scaled matrix using ComplexHeatmap. Clonotypes were called according to their TCR

488 amino acid sequence. Gene Set Enrichment Analysis of the top seven dominant clonotypes was

489 performed on the log Fold Change values from Seurat::FindMarkers () comparing the inflamed to

490 the excluded phenotypes using gseGO() function of the ClusterProfiler R package on the $\mathrm{CC}$

491 Ontology collection.

492

\section{D in vitro tumoroid cultures/co-cultures}

KPP murine pancreatic cancer cells expressing human HER2 and cytosolic GCaMP6 were

495 suspended in 3-dimensional collagen matrices as described in Geraldo et $\mathrm{al}^{37}$. In short, a solution

496 of rat-tail collagen I (MilliporeSigma, MA, USA) was brought to a neutral $\mathrm{pH}$ on ice and mixed

497 with KPP.hHer2.GCaMP6 cells to a final concentration of $2 \mathrm{mg} / \mathrm{mL}$ collagen and $1.0 \times 10^{4}$ cells.

$498150 \mu \mathrm{L}$ of this suspension were added to individual wells of a 8 chambered cover glass (Cellvis,

$499 \mathrm{CA}, \mathrm{USA})$. The chambers were incubated at $20-25^{\circ} \mathrm{C}$ for $15 \mathrm{~min}$ then incubated at $37^{\circ} \mathrm{C}$ with $5 \%$

$500 \mathrm{CO}_{2}$ for an additional $15 \mathrm{~min}$. After incubation, $500 \mu \mathrm{L}$ of complete media containing Gibco RPMI

5011640 (Thermo Fisher Scientific, MA, USA) with 10\% Gibco fetal calf serum (Thermo Fisher

502 Scientific, MA, USA), $2 \mathrm{mM}$ L-glutamine, and $50 \mathrm{U} / \mathrm{mL}$ Gibco penicillin/streptomycin (Thermo 
503 Fisher Scientific, MA, USA) was carefully added to each well. Cells were allowed to grow in 504 collagen matrices for 5 days prior to imaging.

505 3D imaging of OT-I T cells interacting with KPP.hHer2.GCaMP6 tumoroids in collagen 506 matrices was performed on a TiE microscope (Nikon, Japan) with CSU-X1 Spinning Disk 507 (Yokogawa Electric, Japan) and Prime sCMOS camera (Photometrics, AZ, USA). The media in 8 508 chamber imaging slides containing tumor cell-collagen matrices was replaced with Gibco RPMI 5091640 with no phenol red (Thermo Fisher Scientific, MA, USA) with $10 \%$ Gibco fetal calf serum 510 (Thermo Fisher Scientific, MA, USA), $2 \mathrm{mM}$ L-glutamine, and $50 \mathrm{U} / \mathrm{mL}$ Gibco 511 penicillin/streptomycin (Thermo Fisher Scientific, MA, USA) with $3 \mu \mathrm{M}$ of propidium iodide

512 (Thermo Fisher Scientific, MA, USA). OT-I cells were pre-labeled with Celltrace FarRed (Thermo

513 Fisher Scientific, MA, USA) according to manufacturer's protocol. FarRed-labeled OT-I cells

514 were added to each chamber containing collagen-suspended KPP.hHer2.GCaMP6 tumoroids and 515 allowed to infiltrate for 2 hours prior to imaging. At the time of imaging, T-cell-dependent 516 bispecific antibody (anti-hHer2::anti-CD3e) was added to the chambers at 500nM final 517 concentration to induce CTL recognition of hHer2-expressing cancer cells. In control conditions, 518 no antibody was added.

520 STAMP microtumor correlative imaging of $\mathrm{Ca}^{2+}$ influx and $\mathrm{T}$ cell infiltration

RAG-2-deficient mice with adoptively transferred tdTomato + CD3 $+\mathrm{T}$ cells bearing 522 mTagBFP2 and GCaMP6-expressing KPP STAMP microtumors were anaesthetized by isoflurane 523 inhalation to effect and imaged daily from 4 days post tumor implantation to 15 days post tumor 524 implantation at the same ear regions. Epifluorescence time-lapse microscopy image series are 525 acquired daily at the same ear regions with a 1.0x Leica PlanApo objective (Leica 10450028) on 
526 a Leica M205 FA epifluorescence stereomicroscope every minute for 60-70 min. Image analyses

527 was performed using Imaris software (Bitplane, United Kingdom). Time-lapse image series of 528 individual tumors at 8 days post tumor implantation are semi-automatically segmented and 529 analyzed for $\mathrm{Ca}^{2+}$ influx between time points. In addition, tumor sizes, $\mathrm{T}$ cell abundances and $\mathrm{T}$ 530 cell infiltrations are analyzed. Time-lapse image sequences of individual tumors at 13 days post 531 tumor implantation are semi-automatically segmented and are analyzed for tumor size to determine 532 Day8-to-Day13 tumor growth.

534 STAMP microtumor correlative imaging of $\mathrm{Ca}^{2+}$ influx and $\mathrm{T}$ cell infiltration upon TDB administration

FOXN1-deficient nude mice with adoptively transferred tdTomato $+\mathrm{CD} 3+\mathrm{T}$ cells bearing

538 inhalation to effect and imaged 12 days post tumor implantation. Image series are acquired every

53990 seconds for 45 min with a two-photon laser-scanning microscope (Ultima In Vivo Multiphoton

540 Microscopy System, Bruker Technologies, MA, USA) with alternating excitation from dual

541 Ti:sapphire lasers (MaiTai DeepSee, Spectra Physics, MA, USA) tuned to $830 \mathrm{~nm}$ and $980 \mathrm{~nm}$,

542 and a 16x numerical aperture 0.8 immersion objective lens (Nikon, Japan). Thereafter, $\mathrm{T}$ cell-

543 dependent bispecific antibodies (anti-hHer2::anti-CD3e) are administered intravenously and

544 multiphoton time-lapse microscopy image acquisition is continued at the same region. Time-lapse 545 image series of individual tumors are semi-automatically segmented with Imaris software 546 (Bitplane, United Kingdom) and analyzed for $\mathrm{Ca}^{2+}$ influx between time points.

\section{$548 \quad$ Image analysis}


$549 \mathrm{Ca}^{2+}$ influx index in vivo epifluorescence microscopy: An isosurface is created that matches the

550 tumor-associated mtagBFP-2 fluorescence of individual STAMP microtumors. The sum of

551 mtagBFP2 and GCaMP6 fluorescence pixel intensities are calculated for each channel for the

552 tumor isosurface for each time point. The absolute delta of the sum fluorescence intensities

553 between consecutive time points are calculated, averaged for each fluorophore and normalized by

554 the respective mean MFI. The $\mathrm{Ca}^{2+}$ influx index is the result of dividing the normalized average

555 delta sum of GCaMP6 intensities by the normalized average delta sum of mtagBFP2 intensities.

557 T cell abundance: An isosurface is created that matches the tumor-associated mTagBFP2

558 fluorescence of individual STAMP microtumors. The mean fluorescence intensities (MFIs) for the

559 T cell-associated tdTomato fluorescence are determined for the tumor isosurface for each time

560 point. The $\mathrm{T}$ cell abundance index is the result of calculating the median of the tdTomato MFIs

561 across all time points.

562

563 T cell infiltration index: An isosurface is created that matches the tumor-associated mTagBFP2

564 fluorescence of individual STAMP microtumors. Using the tumor isosurface, two new regions are

565 defined: the tumor center (central $50 \%$ of tumor isosurface) and the tumor periphery (area

566 surrounding the tumor that is up to $50 \mu \mathrm{m}$ distance from tumor border). The MFIs for the T cell-

567 associated tdTomato fluorescence are determined for the tumor center and the tumor periphery for

568 each time point and the median of MFIs across all time points is calculated. The T cell infiltration

569 index is the result of the ratio of those medians (center/periphery). 
$571 \mathrm{Ca}^{2+}$ influx index in vivo 2-photon microscopy: An isosurface is created that matches the tumor-

572 associated mtagBFP-2 fluorescence of individual STAMP microtumors. The sum of mTagBFP2

573 and GCaMP6 fluorescence pixel intensities are calculated for the tumor isosurface for each time

574 point. The absolute delta of the sum fluorescence intensities between consecutive time points are

575 calculated, averaged for each fluorophore and normalized by the respective mean MFI across all

576 time points. The normalized average delta sum of GCaMp6 intensities is divided by the normalized

577 average delta sum of mTagBFP2 intensities (value1). Also, the average standard deviation of

578 mTagBFP2 and GCaMP6 fluorescence of every pixel of the tumor isosurface is calculated across

579 the time series. The average standard deviation of GCaMP6 is divided by the average standard

580 deviation of mTagBFP2 (value2). The $\mathrm{Ca}^{2+}$ influx index (2-photon) is the result of multiplying

581 value1 and value2.

$583 \mathrm{Ca}^{2+}$ influx index in vitro spinning disk confocal microscopy: An isosurface is created that matches

584 the tumor-associated GCaMP6 background fluorescence of tumor cells. The median of GCaMP6

585 MFIs are calculated for the tumor cell isosurfaces for each time point. The absolute delta of the

586 median MFIs between consecutive time points are calculated and averaged. The $\mathrm{Ca}^{2+}$ influx index

587 (spinning disk confocal) is the result of dividing the average delta median GCaMP6 MFIs by the

588 mean GCaMP6 MFI across all time points.

$590 \quad$ PI influx index in vitro spinning disk confocal microscopy: An isosurface is created that matches

591 the tumor-associated GCaMP6 background fluorescence of tumor cells. The median of propidium

592 iodide MFIs are calculated for the tumor cell isosurfaces for each time point. The absolute delta of

593 the median MFIs between consecutive time points are calculated and averaged. The propidium 
594 iodide influx index is the result of dividing the average delta median propidium iodide MFIs by

595 the mean propidium iodide MFI across all time points.

596

597 U-net model training: Images of tumor fluorescence are binned and resized to $512 \times 512$ using

598 custom FIJI scripts. Binary (2-class) masks are manually generated with $1=$ Tumor, $0=$ Background.

599 A TensorFlow U-net model adapted from https://github.com/zhixuhao/unet was trained on a 600 dataset of 595 paired images with masks (70\% training and 30\% validation) for 7 epochs until the 601 model began to overfit as indicated by the training accuracy exceeding the validation accuracy 602 without improving loss.

604 Image segmentation, tracking: Images of tumor fluorescence are binned and resized to $512 \times 512$ 605 using custom FIJI scripts. Initial segmentation guesses are generated by applying the trained U606 net using TensorFlow. Custom FIJI scripts are used to un-bin tumor segmentation to restore 607 original size and resolution, enable manual review and editing of all tumor segmentation masks, 608 and manually track tumors through multiple time points. If a tumor is no longer detectable during 609 the course of an experiment, it is designated a complete responder (C.R.). If a tumor decreases 610 from its maximum size by $20 \%$ or more, it is designated a partial responder (P.R.). Remaining 611 tumors are designated as stable disease (S.D.) and progressing disease (P.D.).

613 T cell quantification: Custom FIJI scripts are used to identify the centroid and Feret diameter of 614 each tumor ROI and determine the median radial fluorescence profile of the $\mathrm{T}$ cell fluorescence 615 channel. Custom Python scripts are used to determine the overall median $\mathrm{T}$ cell fluorescence 616 intensity and categorize radial fluorescence profiles as "desert", "excluded" or "inflamed". Tumors 
617 are classified as "excluded" or "inflamed" using a ratiometric cutoff. If the radial profile within

618 the inner $25 \%$ of the tumor is consistently greater than $60 \%$ of the maximum fluorescence for that

619 tumor it is designated as inflamed. If the radial profile within the inner $25 \%$ of the tumor is

620 consistently less than $40 \%$ of the maximum fluorescence for that tumor it is designated as

621 excluded. A tumor is designated a "desert" if the individual tumor's median T cell fluorescence

622 intensity is less than the 25 percentile of median $\mathrm{T}$ cell fluorescence for all tumors measured on

623 the first imaging day and the radial profile does not indicate an excluded pattern as described

624 above. If a tumor shows an excluded profile based on the ratiometric criteria, but the $\mathrm{T}$ cell

625 intensity at the core of the tumor (inner 25\%) is greater than the median $\mathrm{T}$ cell intensity for all

626 inflamed tumors, it is re-classified as inflamed. If a tumor fails to meet the above ratiometric

627 cutoffs, the phenotype determined the previous day is propagated forward until the next definitive

628 classification.

629

630 Clustering and Markov analysis: Custom python scripts are used to assign an integer value to the

631 T cell phenotype classification with $1=$ desert, $2=$ excluded, $3=$ inflamed. If the mouse is euthanized,

632 the remaining time points are assigned value $=0$. If the tumor resolves, the remaining time points

633 are assigned value $=5$. Tumor trajectories are ordered by hierarchical clustering of tumor phenotype

634 lists. Subsequent heatmaps for tumor area, median T cell infiltration and tumor growth are ordered

635 according to this phenotype clustering. Transition state matrices for Markov analysis are generated

636 using custom python scripts. To assess significance, phenotype states are randomized 10 times

637 using Python random.shuffle() and new transition state matrices are calculated. 
639 T cell trajectory analysis: Custom python scripts are used to calculate an exclusion ratio where the

640 numerator represents the maximum value of the median radial profile, and the denominator is the

641 median intensity at the core of the tumor (inner 25\%). These values are compared between tumors

642 relative to the time-normalized trajectory of each tumor by fitting with a spline (using the SciPy

643 Interpolation sub-package), and then calculating a median value at each time point across all

644 tumors.

645

$646 \quad$ Multidimension analysis

647 Data were normalized and standardized (Z-score) before the SPADE tree was built and the EMD

648 scores were calculated.

649 -SPADE trees (Spanning-tree Progression Analysis of Density-normalized Events)

650 We used the set of four parameters ( $\mathrm{T}$ cell abundance in the tumor core and tumor periphery,

651 exclusion ratio, and tumor size) to build SPADE trees (SPADE

652 V3.0, http://pengqiu.gatech.edu/software/SPADE/) for $\mathrm{n}=300$. Where $\mathrm{n}$ is the number of desired

653 clusters. The total number of tumors $(\sim 11 \mathrm{k})$ were distributed between 300 SPADE tree nodes

654 (details on per node tumor distribution can be found on Extended-Data Fig4.y, bottom, right

655 panel). SPADE views single-object (e.g., cell or tumor) data as a multi-dimensional point cloud

656 and uses topological methods to reveal the geometry of the cloud in 2-dimensional space. Single

657 objects are organized in the 2-dimensional tree structure according to their similarity to each other

658 in the multi-dimensional space. Categorical variables such, e.g. phenotype or response, were

659 converted to the numerical variables that were further used to color the SPADE tree.

660 -EMD scores (Earth Mover's Distance) 
661 We used the FlowJo V10 (FlowJo, LLC) to run the UMAP analysis (with the default input 662 parameters) on all of the day 6-17 data points simultaneously. The UMAPs were built using a set 663 of four parameters ( $\mathrm{T}$ cell abundance in the tumor core and tumor periphery, exclusion ratio, and 664 tumor size). We further converted UMAP plots into the density plots for each treatment group 665 (control, anti-TGF- $\beta$, anti-PD-L1, and combination of anti-PD-L1 with anti-TGF- $\beta$ ) and 666 calculated the EMD scores ${ }^{38}$ between each treatment group and the group of responders (C.R and 667 P.R). EMD measures the distance between two probability distributions, e.g., the control treatment 668 group and responders' group.

\section{Immunophenotypes in imCORE Paired Biopsy trial}

Tumor biopsies were obtained from patients enrolled in the imCORE ${ }^{39}$ Paired Biopsy trial

672 (NCT03333655) between January 2018 and March 2020. This study is an ongoing, open-label,

673 multicenter trial initiated in February 2018 and conducted globally including study centers in the

674 United States, France, and Spain. Adult patients with metastatic cancer or hematological

675 malignancies who demonstrated clinical benefit on CIT and had a tumor biopsy both at baseline 676 (pre-treatment/archival) and at progression were eligible for inclusion. CIT included marketed 677 agents (including those targeting CTLA-4, programmed death-ligand 1 [PD-L1] or programmed

678 death-1 [PD-1]) or those administered through participation in a Roche/Genentech CPI clinical 679 trial. Patients with best overall response (per Response Evaluation Criteria in Solid Tumors version 680 1.1) of complete response (CR), partial response (PR) or stable disease (SD) $>6$ months (or $>3$ 681 months if enrolled under an earlier protocol version) were eligible. PanCK/CD8 dual staining was 682 performed on histologic sections from baseline and progression formalin-fixed paraffin-embedded 
683 tumor samples. Immune phenotypes were determined by a pathologist (Histogenex) using defined 684 criteria $^{40}$.

\section{Statistics and figure preparation}

Statistical analysis was performed by t-test, Mann-Whitney test, one-way ANOVA with

688 Bonferroni's multiple comparison's test or log-rank test. $\mathrm{P}<0.05$ was considered significant.

689 Models in Figs. 1a, 2c, 3g were created using BioRender (https://biorender.com/).

>>>Supplementary Information

\section{Supplementary Videos}

693 1) Example of live in vivo 2photons imaging of an individual KPP BFP+ STAMP tumor showing

694 the migration of TdTomato+ OTI T cells and WT GFP+ T cell.

695 2) Epifluorescence imaging of 3 adjacent KPP tumors (magenta) with excluded, inflamed and 696 desert Immune phenotypes, showing $\mathrm{T}$ cell migration (cyan) in adjacent tumors (related to 697 Extended Fig.2a)

698 3) Confocal imaging followed by 3D reconstruction of a whole cleared lung showing KPP-GFP+ 699 tumor foci and TdTomato+ T cells 8 days after i.v transfer. (related to Fig.2b)

700 4) Confocal live imaging showing low Ca2+ flashes (green) in GCaMP6+ Her2+ KPP organoid, 701 cocultured with T cells (magenta) and propidium Iodide (Red) (related to Fig.3h and Extended $702 \quad$ Fig. $3 p)$

703 5) Confocal live imaging showing high Ca2+ flashes (green) in GCaMP6+ Her2+ KPP organoid, 704 cocultured with $\mathrm{T}$ cells (magenta)and TDB (aHer2/aCD3) to force tumor cell killing by $\mathrm{T}$ cells 
705 (positive control). Propidium Iodide entry (Red) reveals tumor cell death. (related to Fig.3h and 706 Extended Fig.3p)

707 6) In vivo 2 photons live imaging showing absence of $\mathrm{Ca} 2+$ flashes in GCaMP $6+\mathrm{BFP}+\mathrm{Her} 2+\mathrm{KPP}$

708 tumors implanted in Nude mouse (negative control) (Related to Extended Fig.3t)

709 7) In vivo 2photons live imaging showing some Ca2+ flashes in GCaMP6+ BFP + Her2 + KPP

710 tumors implanted in Nude mouse reconstituted with TdTomato $+\mathrm{T}$ cells (related to Extended

711 Fig.3t)

712 8) In vivo 2photons live imaging showing high level of $\mathrm{Ca} 2+$ flashes in GCaMP6 $+\mathrm{BFP}+\mathrm{Her} 2+$

713 KPP tumors implanted in Nude mouse reconstituted with TdTomato + T cells and treated with TDB

714 (aHer2/aCD3) to force tumor cell killing by T cells (positive control) (related to Extended Fig.3t)

715 9) Epifluorescence live imaging of an Immune Inflamed tumor showing high Ca2+ flashes in

716 GCaMP6+ KPP tumor cells implanted in Nude mouse reconstituted with TdTomato $+\mathrm{T}$ cells

717 (related to Fig3j)

718 10) Epifluorescence live imaging of an Immune excluded tumor showing low Ca2+ flashes (green)

719 in GCaMP6+ KPP tumor cells implanted in Nude mouse reconstituted with TdTomato + T cells

$720 \quad$ (related to Fig.3j)

\section{Supplementary Tables}

723 1) Markov Probabilities of Transition between Immune Phenotypes (related to Fig.4h and 724 Extended Fig.4i-j)

725 2) Raw measurements of 14300 KPP-GFP STAMP tumors implanted into RAG2-/- mice 726 reconstituted with TdTomato $+\mathrm{T}$ cells and treated with CIT one Day after tumor implantation 727 (related to Fig.4) 
3) Clinical summary (related to Fig.4q)

>>Acknowledgements

731 We thank members of C. Moussion, S.J. Turley, and I. Mellman laboratories for advice,

732 discussions and reagents; B. Hough \& R. Asuncion, for animal husbandry; R. Garcia-Gonzalez, J.

733 Yamada \& E. Chua for veterinary care; the Genentech FACS group for technical assistance;

734 HyperVoxel, Johannes Schoeneberg and Gautham Raghupathi for the support with Image

735 processing and machine learning and the Genentech postdoctoral program for support. This study

736 was funded by Genentech/Roche.

>>Author contributions

739 G.O.M and M.B. designed and performed the experiments, analyzed and interpreted the data,

740 C.B.C developed the high content image analysis pipeline to analyze tumors features over-time,

741 analyzed and interpreted the STAMP CIT timeseries experiments, J.P.J analyzed and interpreted

742 NGS data, D.O analyzed and interpreted the STAMP CIT timeseries experiments, A.T.R., J.D,

743 S.M, A.Y performed experiments; S.T edited the manuscript, Z.M supervised sequencing, T.W

744 participated in single cell analysis, R.P. supervised the collection of clinical samples; I.M discussed

745 the data and edited the manuscript; Y.W and S.M shared clinical trial data, C.G-R, C.E.A, D.S

746 collected patient samples for imCORE studies, C.M. conceived and supervised the study,

747 interpreted the data and wrote the manuscript; and all authors read and approved the final article. 
750 G.O.M, M.B, C.B.C, J.P.J, A.T.R, D.O, A.Y, J.D, S.T, Y.W, S.M, Z.M, T.W, R.P, I.M and C.M

751 declare that they are Genentech/Roche employees.

752

753 ORCIDs:

754 Catherine B. Carbone: 0000-0003-1610-4691

755 Jonas Doerr: 0000-0003-3599-6023

756 Carlos Gomez-Roca: 0000-0002-8043-2529.

757 Ira Mellman: 0000-0002-6132-7299

758 Christine Moussion: 0000-0002-1641-4094

759 Guadalupe Ortiz-Muñoz: 0000-0002-8928-0576

760 Joaquin Pechuan-Jorge: 0000-0001-8938-7931

761 Alex T. Ritter: 0000-0002-3476-2252

762 Thomas Wu: 0000-0003-4505-4531

763

764 >> References

765

766 1. Chen, D. S. \& Mellman, I. Elements of cancer immunity and the cancer-immune set point.

767 Nature 541, 321-330 (2017).

768 2. Galon, J. \& Bruni, D. Approaches to treat immune hot, altered and cold tumours with

769 combination immunotherapies. Nat Rev Drug Discov 18, 197-218 (2019).

770 3. Galon, J. et al. Type, Density, and Location of Immune Cells Within Human Colorectal Tumors

771 Predict Clinical Outcome. Science 313, 1960-1964 (2006). 
772 4. Bruni, D., Angell, H. K. \& Galon, J. The immune contexture and Immunoscore in cancer

773 prognosis and therapeutic efficacy. Nat Rev Cancer 20, 662-680 (2020).

774 5. Tumeh, P. C. et al. PD-1 blockade induces responses by inhibiting adaptive immune resistance.

$775 \quad$ Nature 515, 568-571 (2014).

776 6. Gibney, G. T., Weiner, L. M. \& Atkins, M. B. Predictive biomarkers for checkpoint inhibitor-

777 based immunotherapy. Lancet Oncol 17, e542-e551 (2016).

778 7. Herbst, R. S. et al. Predictive correlates of response to the anti-PD-L1 antibody MPDL3280A

779 in cancer patients. Nature 515, 563-567 (2014).

780 8. Gajewski, T. F. The Next Hurdle in Cancer Immunotherapy: Overcoming the Non-T-Cell-

781 Inflamed Tumor Microenvironment. Semin Oncol 42, 663-671 (2015).

782 9. Bachhav, Y. G., Heinrich, A. \& Kalia, Y. N. Controlled intra- and transdermal protein delivery 783 using a minimally invasive Erbium:YAG fractional laser ablation technology. Eur J Pharm

784 Biopharm 84, 355-364 (2013).

785 10. Chung, W.-J. et al. Kras mutant genetically engineered mouse models of human cancers are 786 genomically heterogeneous. Proc National Acad Sci 114, E10947-E10955 (2017).

787 11. Mariathasan, S. et al. TGF $\beta$ attenuates tumour response to PD-L1 blockade by contributing to 788 exclusion of T cells. Nature 554, 544-548 (2018). 
789 12. Dominguez, C. X. et al. Single-Cell RNA Sequencing Reveals Stromal Evolution into

790 LRRC15+ Myofibroblasts as a Determinant of Patient Response to Cancer Immunotherapy.

791 Cancer Discov 10, 232-253 (2020).

792 13. Desbois, M. et al. Integrated digital pathology and transcriptome analysis identifies molecular 793 mediators of T-cell exclusion in ovarian cancer. Nat Commun 11, 5583 (2020).

794 14. Spranger, S., Bao, R. \& Gajewski, T. F. Melanoma-intrinsic $\beta$-catenin signalling prevents anti795 tumour immunity. Nature 523, 231-235 (2015).

796 15. Spranger, S. \& Gajewski, T. F. Impact of oncogenic pathways on evasion of antitumour 797 immune responses. Nat Rev Cancer 18, 139-147 (2018).

798 16. Liu, X. \& Peng, G. Mitochondria orchestrate T cell fate and function. Nat Immunol 22, 276$799278(2021)$

800 17. Buck, M. D. et al. Mitochondrial Dynamics Controls T Cell Fate through Metabolic 801 Programming. Cell 166, 63-76 (2016).

802 18. Li, W. \& Zhang, L. Rewiring Mitochondrial Metabolism for CD8+ T Cell Memory Formation 803 and Effective Cancer Immunotherapy. Front Immunol 11, 1834 (2020).

804 19. Araki, K. et al. Translation is actively regulated during the differentiation of CD8+ effector T 805 cells. Nat Immunol 18, 1046-1057 (2017).

806 20. Chen, T.-W. et al. Ultrasensitive fluorescent proteins for imaging neuronal activity. Nature 807 499, 295-300 (2013). 
808 21. Tauriello, D. V. F. et al. TGF $\beta$ drives immune evasion in genetically reconstituted colon cancer

809 metastasis. Nature 554, 538-543 (2018).

810 22. Angelova, M. et al. Evolution of Metastases in Space and Time under Immune Selection. Cell

811 175, 751-765.e16(2018).

812 23. Doerr, J. et al. Whole-brain 3D mapping of human neural transplant innervation. Nat Commun

$813 \mathbf{8}, 14162(2017)$

814 24. Schwarz, M. K. et al. Fluorescent-Protein Stabilization and High-Resolution Imaging of

815 Cleared, Intact Mouse Brains. Plos One 10, e0124650 (2015).

816 25. Li, H. \& Durbin, R. Fast and accurate short read alignment with Burrows-Wheeler transform.

817 Bioinformatics 25, 1754-1760 (2009).

818 26. DePristo, M. A. et al. A framework for variation discovery and genotyping using next819 generation DNA sequencing data. Nat Genet 43, 491-498 (2011).

820 27. Saunders, C. T. et al. Strelka: accurate somatic small-variant calling from sequenced tumor821 normal sample pairs. Bioinformatics 28, 1811-1817 (2012).

822 28. Huber, W. et al. Orchestrating high-throughput genomic analysis with Bioconductor. Nat 823 Methods 12, 115-121 (2015).

824 29. Frankish, A. et al. GENCODE reference annotation for the human and mouse genomes. $825 \quad$ Nucleic Acids Res 47, gky955- (2018). 
826 30. Law, C. W., Chen, Y., Shi, W. \& Smyth, G. K. voom: precision weights unlock linear model

827 analysis tools for RNA-seq read counts. Genome Biol 15, R29 (2014).

828 31. Liberzon, A. et al. The Molecular Signatures Database Hallmark Gene Set Collection. Cell 829 Syst 1, 417-425 (2015).

830 32. Gu, Z., Eils, R. \& Schlesner, M. Complex heatmaps reveal patterns and correlations in 831 multidimensional genomic data. Bioinformatics 32, 2847-2849 (2016).

832 33. Butler, A., Hoffman, P., Smibert, P., Papalexi, E. \& Satija, R. Integrating single-cell 833 transcriptomic data across different conditions, technologies, and species. Nat Biotechnol 36, 411$834420(2018)$

835 34. Henningsson, R., Moratorio, G., Bordería, A. V., Vignuzzi, M. \& Fontes, M. DISSEQT836 DIStribution-based modeling of SEQuence space Time dynamics. Virus Evol 5, vez028 (2019).

837 35. Zappia, L. \& Oshlack, A. Clustering trees: a visualisation for evaluating clusterings at multiple 838 resolutions. Gigascience 7, giy083-(2018).

839 36. Borcherding, N., Bormann, N. L. \& Kraus, G. scRepertoire: An R-based toolkit for single-cell 840 immune receptor analysis. F1000research 9, 47 (2020).

841 37. Geraldo, S., Simon, A. \& Vignjevic, D. M. Revealing the Cytoskeletal Organization of 842 Invasive Cancer Cells in 3D. J Vis Exp e50763 (2013) doi:10.3791/50763.

843 38. Orlova, D. Y. et al. Earth Mover's Distance (EMD): A True Metric for Comparing Biomarker 844 Expression Levels in Cell Populations. Plos One 11, e0151859 (2016). 
845 39. Helou, S. M. et al. A relationship-based approach to improving clinical trials: The imCORE

846 research network experience. Contemp Clin Trials 86, 105861 (2019).

847 40. Powles, T. et al. Clinical efficacy and biomarker analysis of neoadjuvant atezolizumab in

848 operable urothelial carcinoma in the ABACUS trial. Nat Med 25, 1706-1714 (2019). 


\section{Fig. 1 Local T cell-mediated rejection of clonal skin tumor array}

a
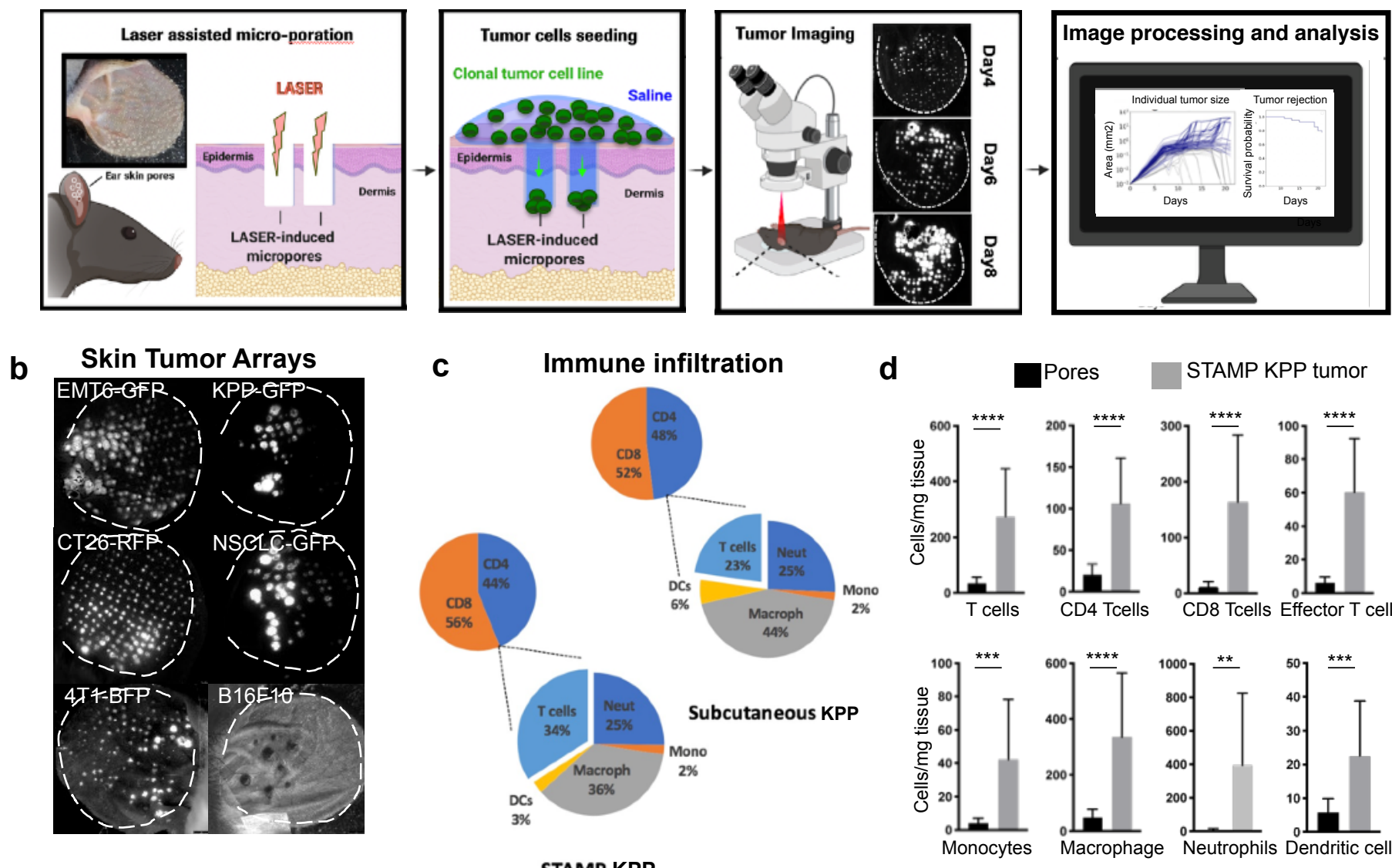

STAMP KPP
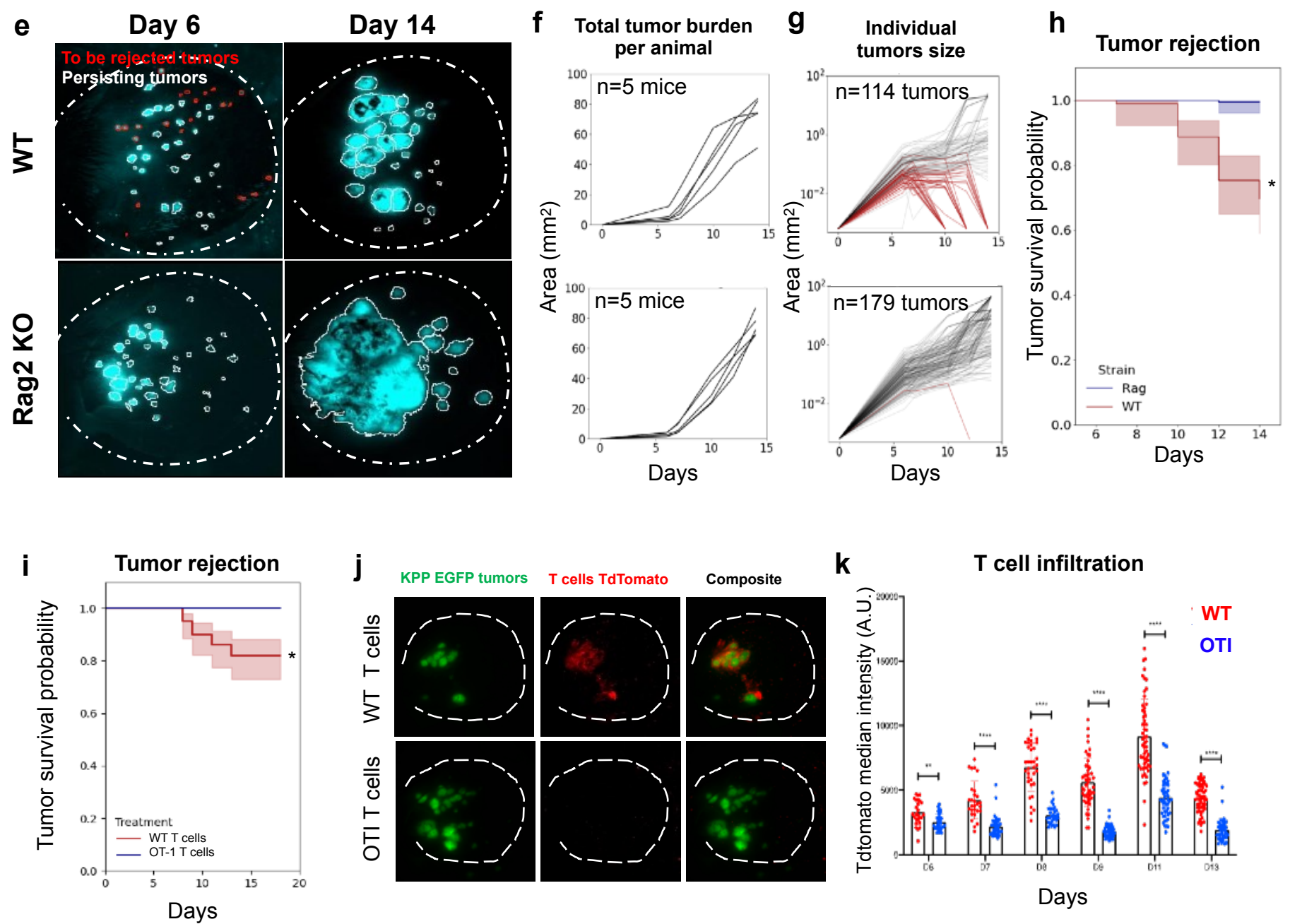

k
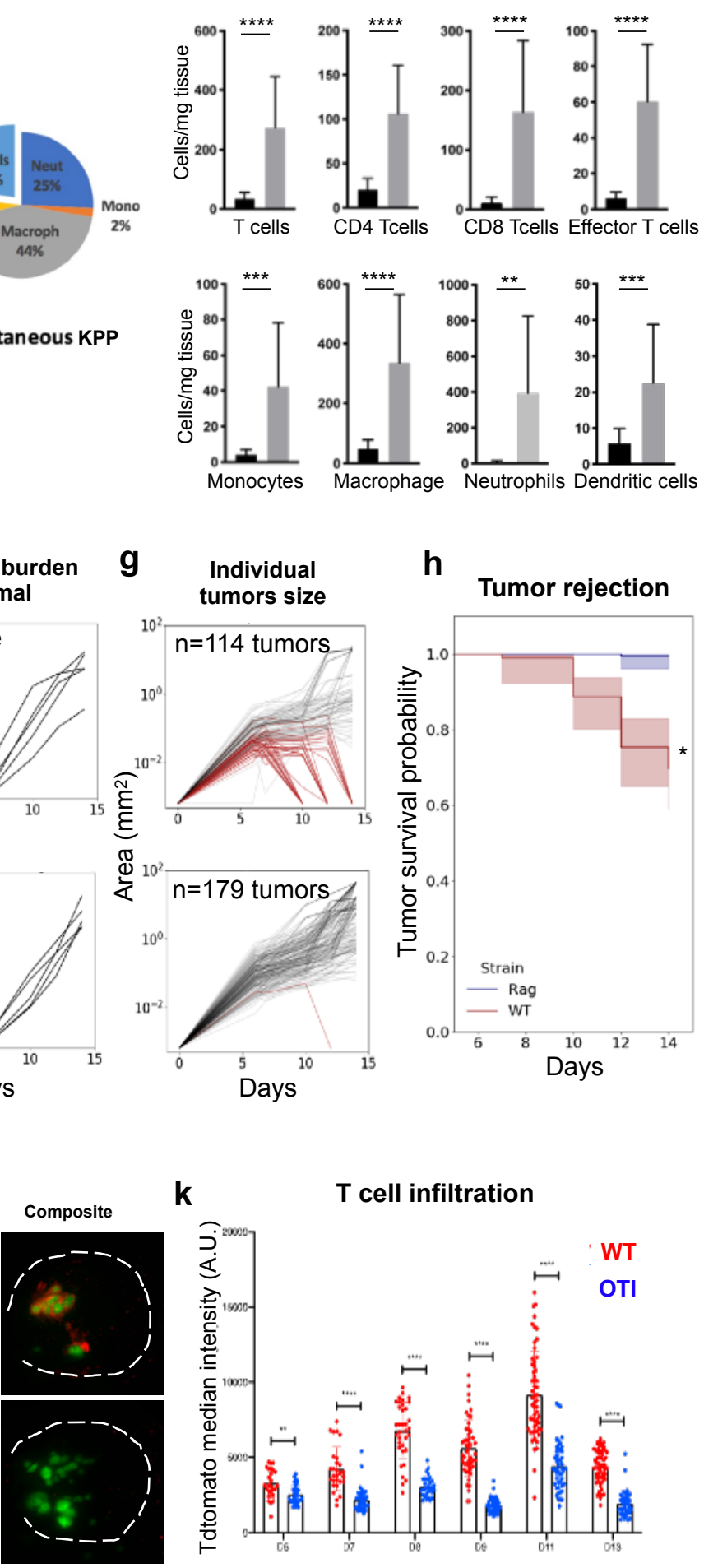

Days 
Fig1. Local T cell-mediated rejection of clonal skin tumor array

a, STAMP (skin țumor a array by $\underline{m}$ icroporation) workflow. Skin microporation with P.L.E.A.S.E.® Laser Device and subsequent seeding of tumor cell suspension. Individual tumors are longitudinally tracked using epifluorescence microcopy and growth kinetics are analyzed by automated computation. b, Representative STAMP tumor arrays of different mouse tumor cell lines at 7 days post tumor implantation for EMT6-GFP, CT26-RFP, 4T1-BFP, KPP-GFP, NSCLC-GFP and 20 days post tumor implantation for B16F10. c, Lymphoid and myeloid immune cell profiling of STAMP tumor array and subcutaneous tumors of KPP-EGFP cancer cells. Tumors were harvested18 days post tumor implantation and pooled. $n=6$ ears, $n=3$ mice for STAMP. $n=5$ mice for subcutaneous tumors. $\mathbf{d}$, Lymphoid and myeloid immune cell profiling of PBS or KPP-EGFP-seeded micropores. STAMP tumor arrays were harvested 18 days post tumor implantation and pooled. $n \geqq 16$ ears, $n=3$ mice. e, Representative STAMP tumor arrays of KPP-EGFP in C57BI6 WT (upper panel) or C57BI6 Rag2 KO (lower panel) mice at 6 and 14 days post tumor implantation. Red encircled tumors are rejected and white encircled tumors are persistent between time points. $n=5$ animals. f, Automated analysis of growth kinetics of total tumor array area $\left(\mathrm{mm}^{2}\right)$ per animal as described in (e) $n=5$ animals. g. Automated analysis of growth kinetics of individual tumor area $\left(\mathrm{mm}^{2}\right)$ as described in (e-f). $n \geqq 114$ tumors, 5 animals pooled. Red lines indicate tumors that are rejected, gray lines indicate tumors that persist. h, Kaplan Meier survival curves of individual tumors as described in (e-g). $n \geqq 114$ tumors, 5 animals pooled. (Shaded area $=95 \%$ confidence interval, log-rank test $p$-value $=2.8 \times 10^{\wedge}-15$ ). $\mathbf{i}$, Kaplan Meier survival curves of individual tumors of KPP-EGFP in RAG-2-deficient C57BL/6J mice reconstituted by adoptive transfer of CD3+ T TdTomato+ T cells from either C57BL/6J WT $(n \geqq 100)$ or OT-1+ mice $n \geqq 88$ tumors. (Shaded area $=95 \%$ confidence interval, log-rank test $p$ -

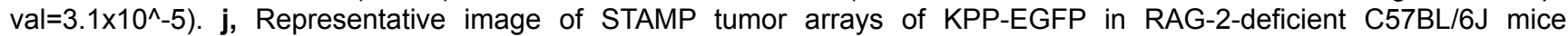
reconstituted by adoptive transfer with CD3+ T cells from either C57BL/6J WT (upper panel) or OT-1+ CD4-cre tdTomato+ (lower panel) mice at 9 days post tumor implantation. $\mathbf{k}$, T cell infiltration kinetics of individual tumors measured by tdTomato MFI of mice described in (i). $n \geqq 26$ tumors. 


\section{Fig. 2 | Heterogeneity and clinical relevance of mouse STAMP tumor-Immune phenotypes}

a

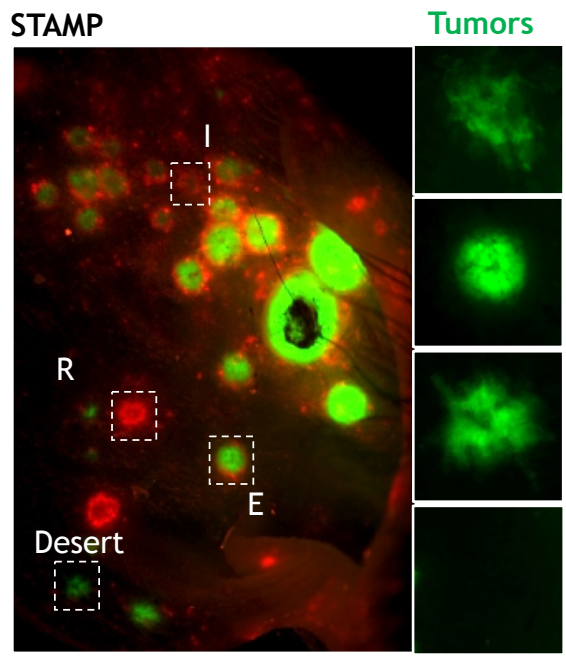

C
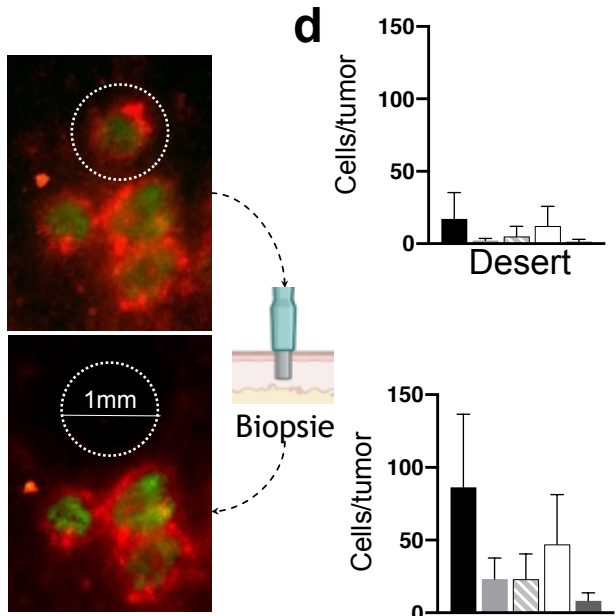

Desert Vs E\&I

$\mathbf{f}$

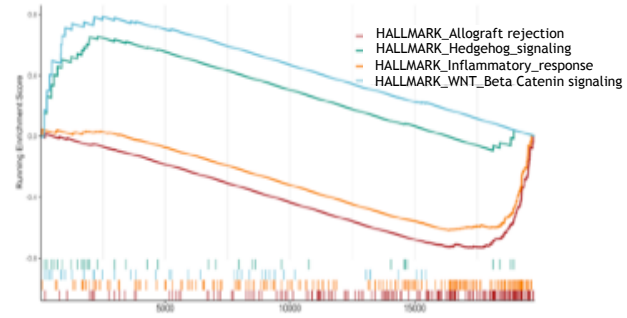

Excluded Vs D\&I

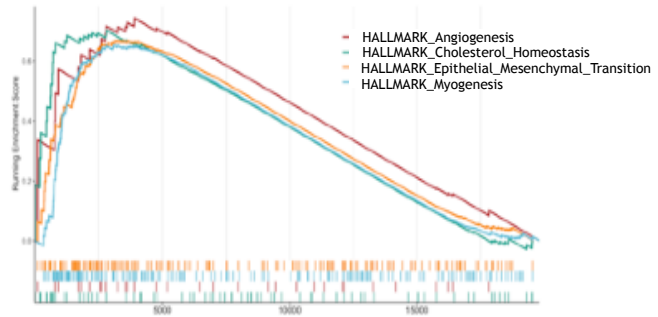

Inflamed Vs E\&D

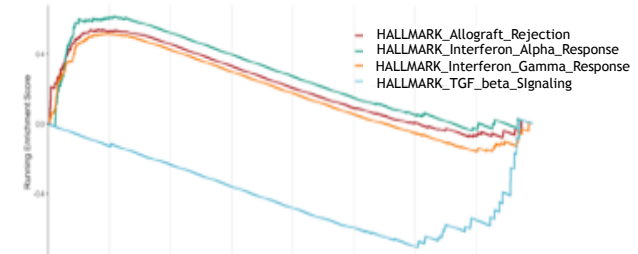

b Lung

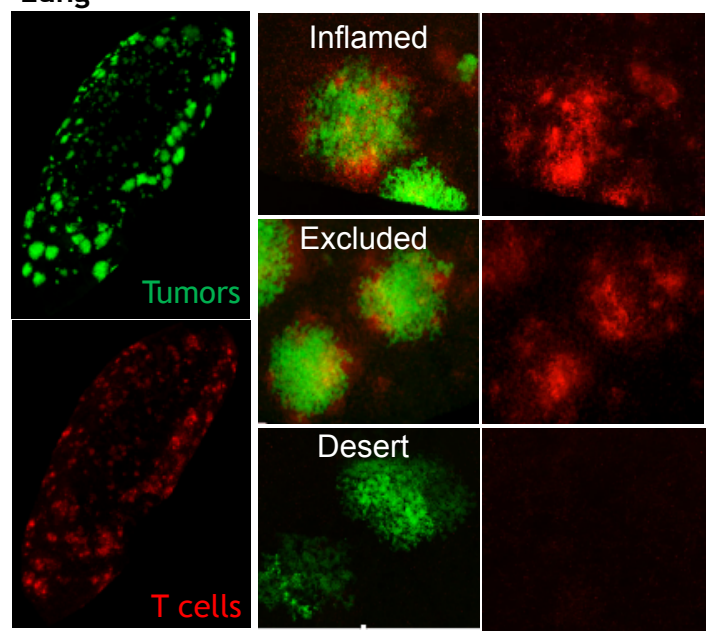

e
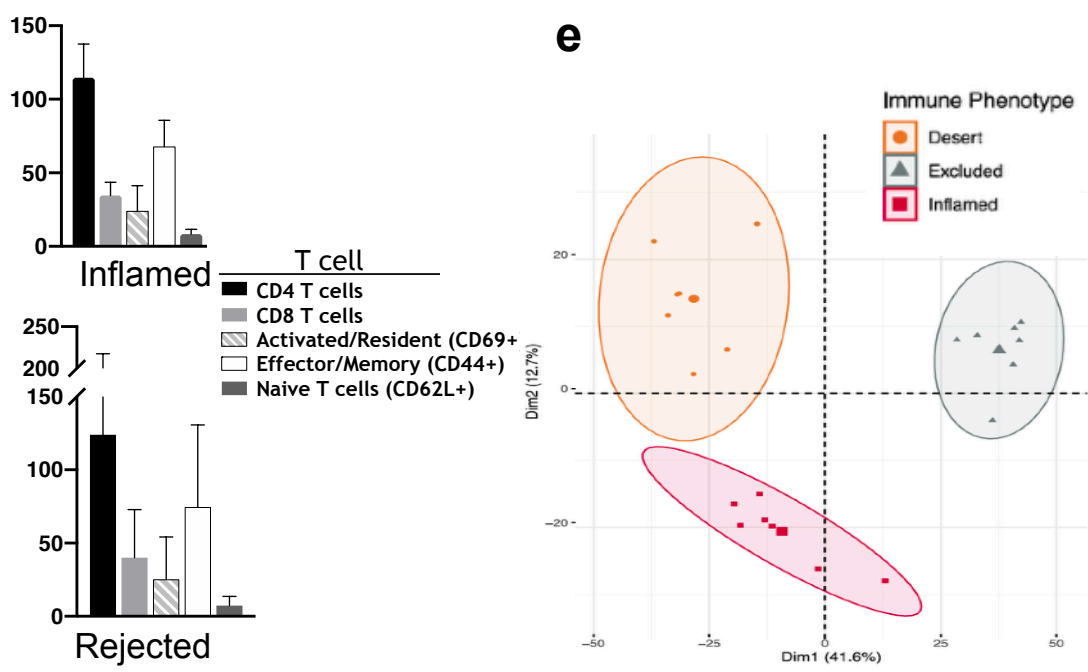

g

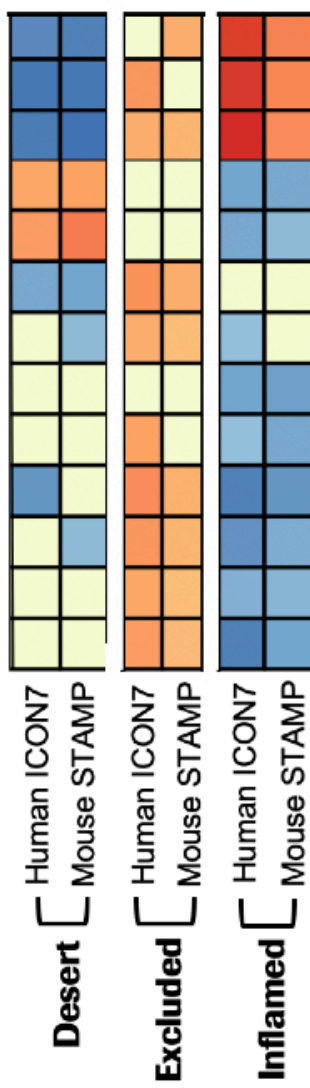

INTERFERON ALPHA RESPONSE ALLOGRAFT REJECTION INTERFERON GAMMA RESPONSE HEDGEHOG SIGNALING WNT BETA CATENIN SIGNALING ANGIOGENESIS HYPOXIA NOTCH SIGNALING TGF BETA SIGNALING EPITHELIAL MESENCHYMAL TRANSITION MYOGENESIS APICAL JUNCTION UV RESPONSE DN 
Fig. 2 | Heterogeneity and clinical relevance of mouse STAMP tumor-Immune phenotypes

a, Representative image of STAMP array of KPP-EGFP implanted in C57BL/6J RAG-2-deficient mice at 8 days post tumor implantation, reconstituted by adoptive transfer of CD3+ T cells from C57BL/6J WT CD4-cre tdTomato+ mice (T cells shown in red, KPP-EGFP shown in green). Left panel represents an overview of the entire ear, right panels are enlarged images of individual tumors with diverse immune phenotypes: inflamed, excluded, desert, resolved. $n=50$ animals. b, Representative experimental lung metastases of KPP-EGFP in C57BL/6J RAG-2-deficient mice at 8 days post tumor implantation, reconstituted by adoptive transfer of CD3+ T cells from C57BL/6J WT CD4-cre tdTomato+ mice (T cells shown in red, KPP-EGFP shown in green). Left panel represents an overview of the entire lung lobe, right panels are enlarged images of metastatic foci with diverse immune phenotypes: inflamed, excluded, desert, resolved. $\mathrm{n}=50$ animals. $\mathrm{n}=5$ animals. c, Representative STAMP as described in (a), before and after tumor biopsy with $1 \mathrm{~mm}$ diameter punch biopsy. d, Flow cytometry-based T-lymphoid immune cell profiling of biopsied tumors pooled by immune phenotype at day 10 . $n=6$ animals, $\geqq 3$ pools of tumors. e, PCA of bulk RNAseq of individually biopsied tumors with differential immune phenotypes: inflamed, desert, excluded. f, Detailed GSEA for selected pathways that are significantly enriched between three different immune phenotypes (inflamed, desert, excluded) from STAMP tumors. g, Heat-map comparing the normalized enrichment scores for pathways that are significantly enriched between the three different immune phenotypes (inflamed, desert, excluded) from either the human ICON7 clinical trial or the mouse STAMP tumors. 
Fig. 3. | Immune phenotype determines T cell function regardless of T cell clonotype

a

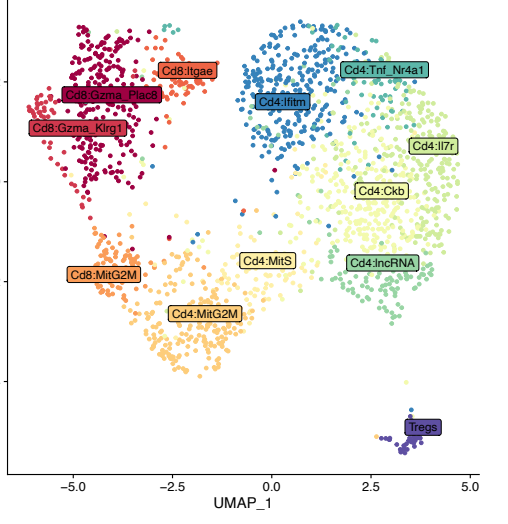

d

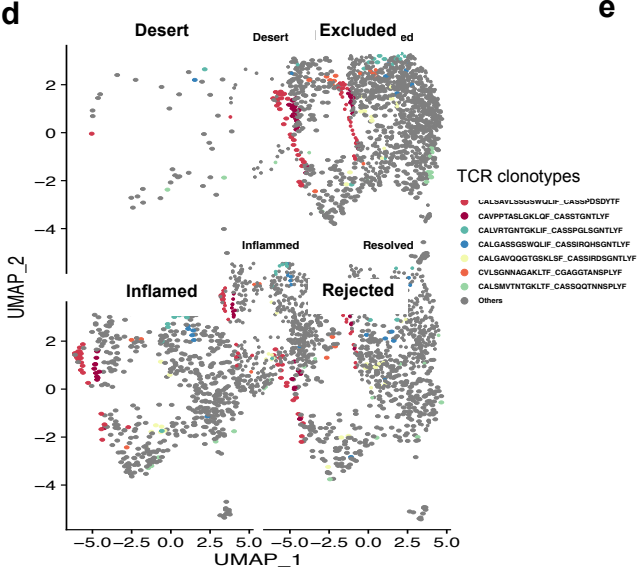

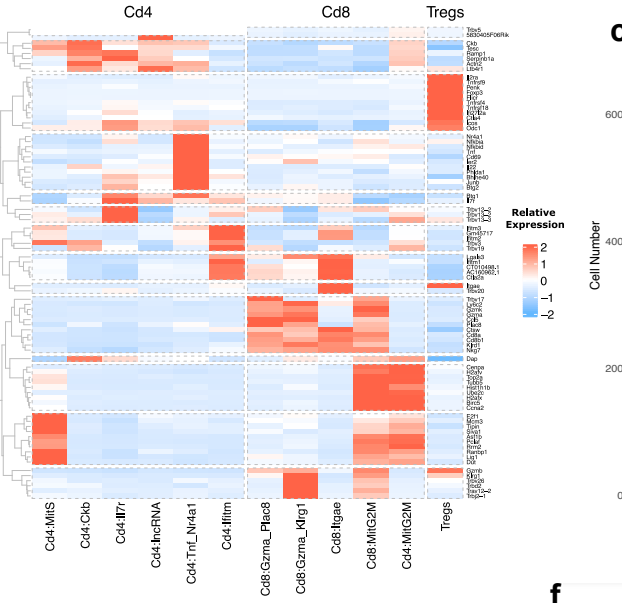

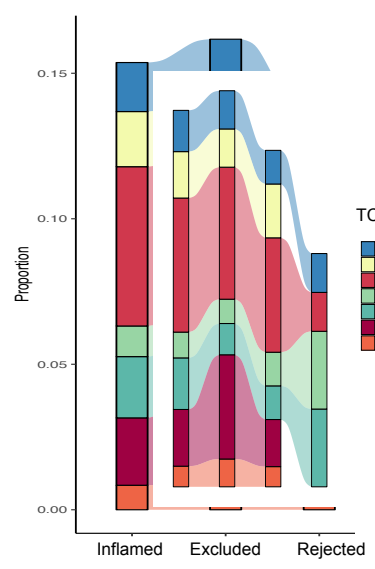

Tumor organoid

f

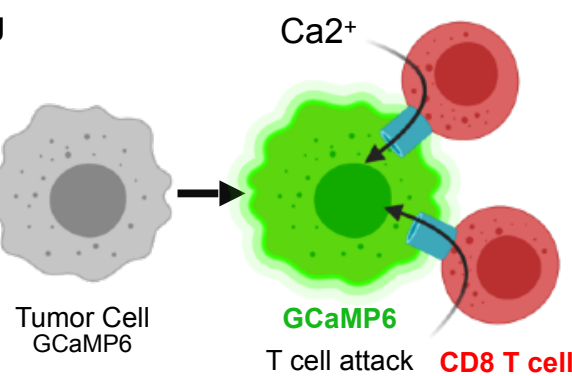

STAMP Inflamed tumor

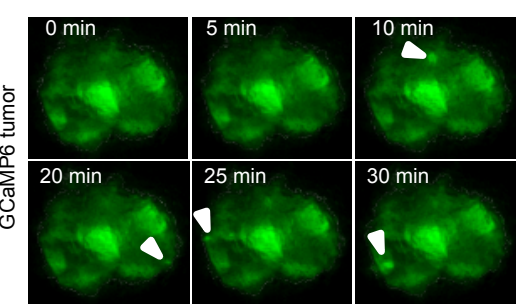

STAMP Excluded tumor

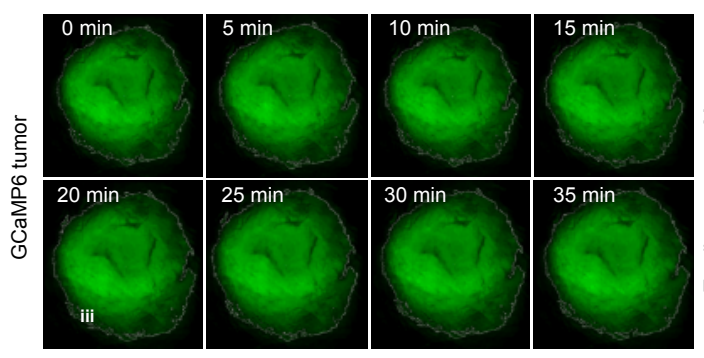

h

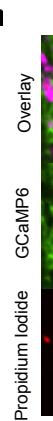

k
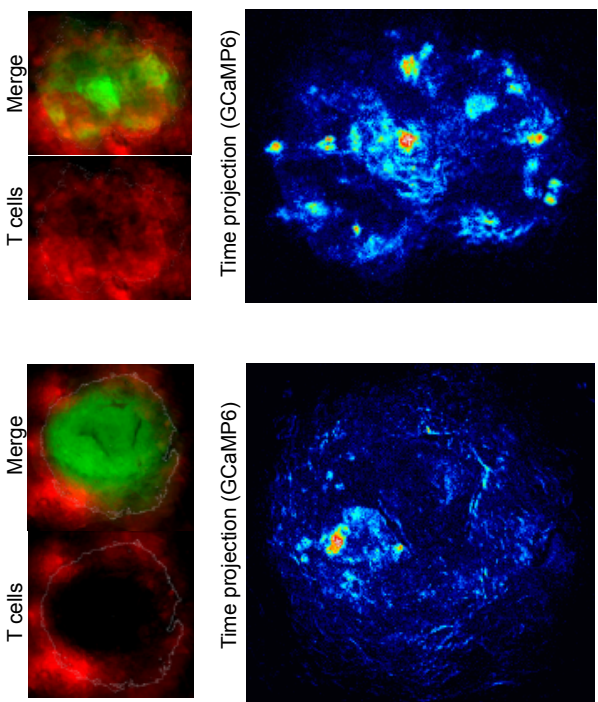
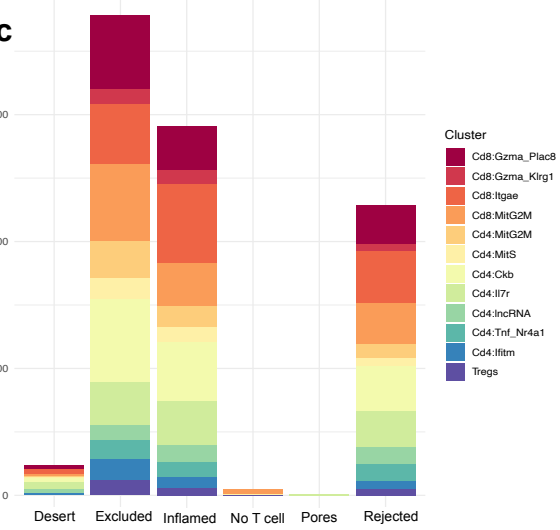

Immune Phenorype

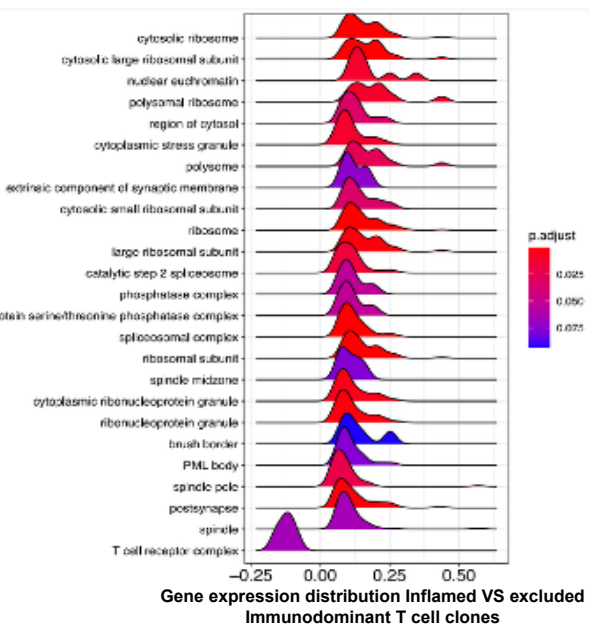
Immunodominant $\mathrm{T}$ cell clones
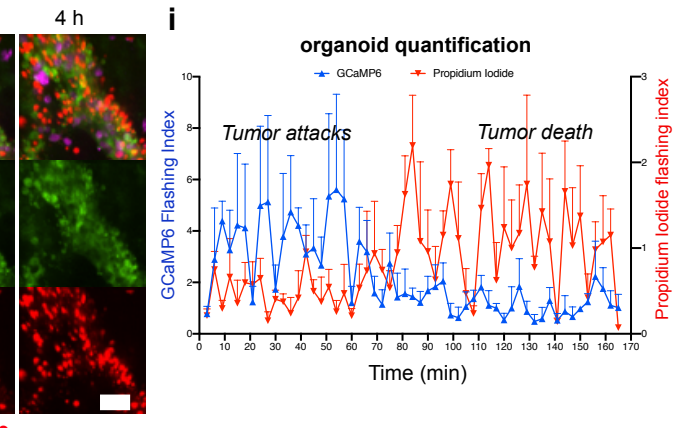

I Total T cell abundance

m Flashing index
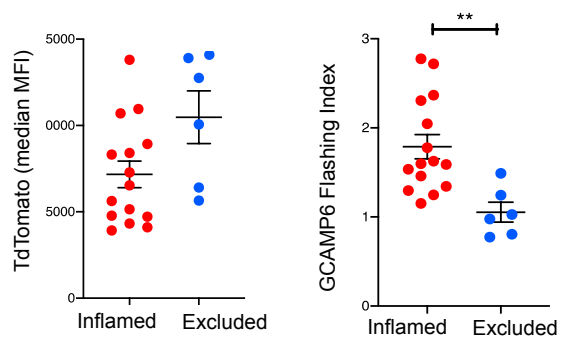

n

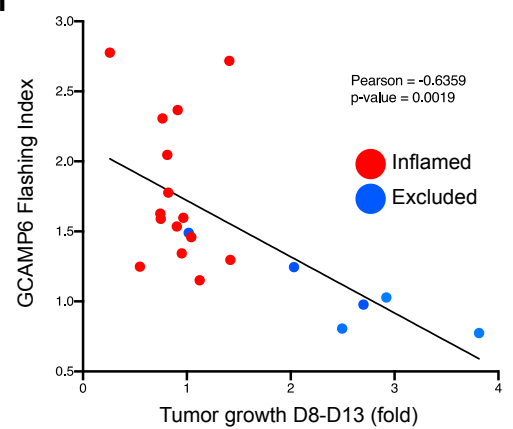


Fig. 3. | Immune phenotype determines T cell function regardless of T cell clonotype

a, UMAP visualization of tdTomato $+T$ cell clusters from pooled STAMP tumor biopsies. $\mathbf{b}$, Heatmap of the average normalized expression of top 10 positive marker genes for each $T$ cell cluster. c, Cell numbers of each $T$ cell subclusters per tumor immune phenotype. d. UMAP visualization of the T cell clusters per tumor immune phenotypes with overlay of the top seven immunodominant clonotypes e, Relative abundance of the top seven immunodominant clonotypes per Immune phenotypes. f, Expression distribution of the core enriched genes for the top 25 enriched pathways comparing immunodominant T cell clones present in Inflamed VS excluded tumors, corrected for their T cell subcluster identity $\mathbf{g}$, cytotoxic $\mathrm{T}$ cells creating $\mathrm{Ca} 2+$ permeable perforin pores in the tumor cell membrane leading to tumor cell killing. Ca2+ influx activates green fluorescence of GCaMP6 calcium sensor. h, Representative in vitro timelapse images of GCaMP6-expressing tumor organoids under T cell attack. (i) Composite of GCaMP6 (green), propidium iodide (red) and T cell fluorescence (magenta) (ii) single channel of GCaMP6 fluorescence, (iii) single channel of propidium iodide fluorescence, $n=3$. $\mathbf{i}$, quantification of calcium flashes and propidium iodide accumulation during T cell-mediated tumor cell killing of organoids. $n=3$. j, Left panel- Representative in vivo time-lapse images of GCaMP6-expressing inflamed (upper panel) or excluded (lower panel) STAMP tumors, as described in (g). Right panelcomposite of tumor and T cell fluorescence to highlight tumor Immune phenotype. GCaMP6 tumor: green and tdTomato T cell: red $\mathbf{k}$, Time projection of maximum minus mean GCaMP6 fluorescence of the tumor described in (j) I, Total T cell abundance (including adjacent area) of individual inflamed and excluded tumors from GCaAMP6 expressing KPPSTAMP arrays $n \geqq 6$. * $p$-value $\leqq 0.05$, unpaired two-sided T-test. $\mathbf{m}, \mathrm{Ca} 2+$ influx indices of inflamed and excluded tumors described in (I). $\mathrm{n} \geqq 6$. ** $\mathrm{p}$-value $\leqq 0.01$, unpaired two-sided T-test. $\mathbf{n}$, Correlation analysis of flashing indices and tumor growth fold change (Day8-to-Day13) of inflamed and excluded tumors described in (I). 
Fig. 4 | Early transition to an inflamed phenotype predicts response to Immunotherapy
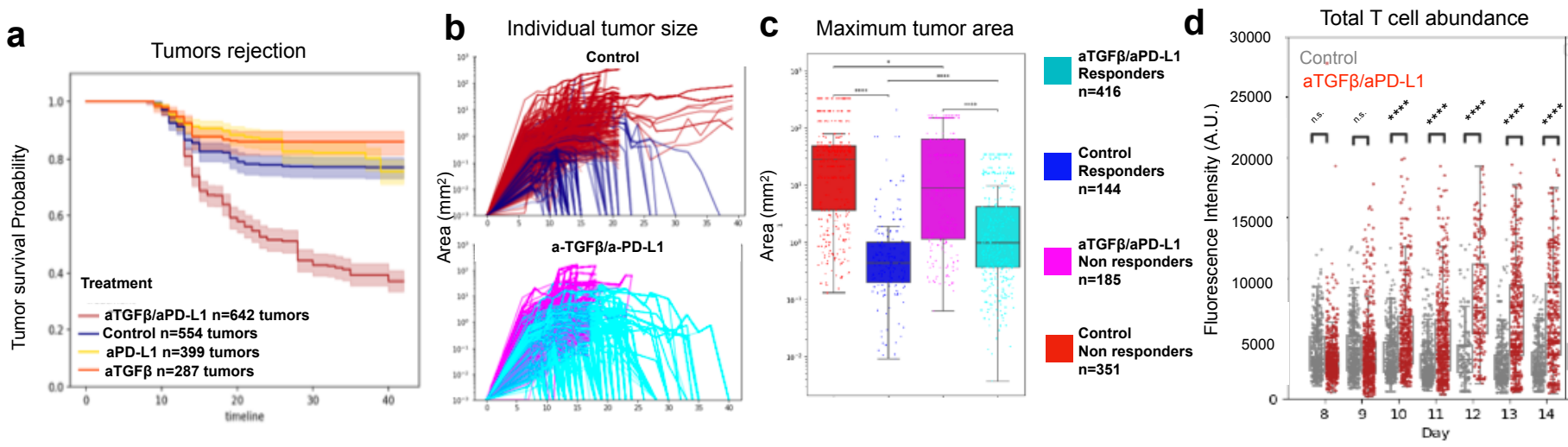

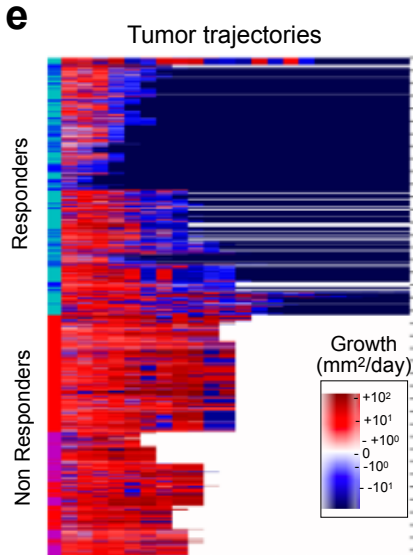

Days

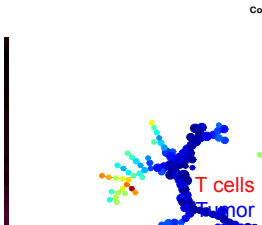

Day 15
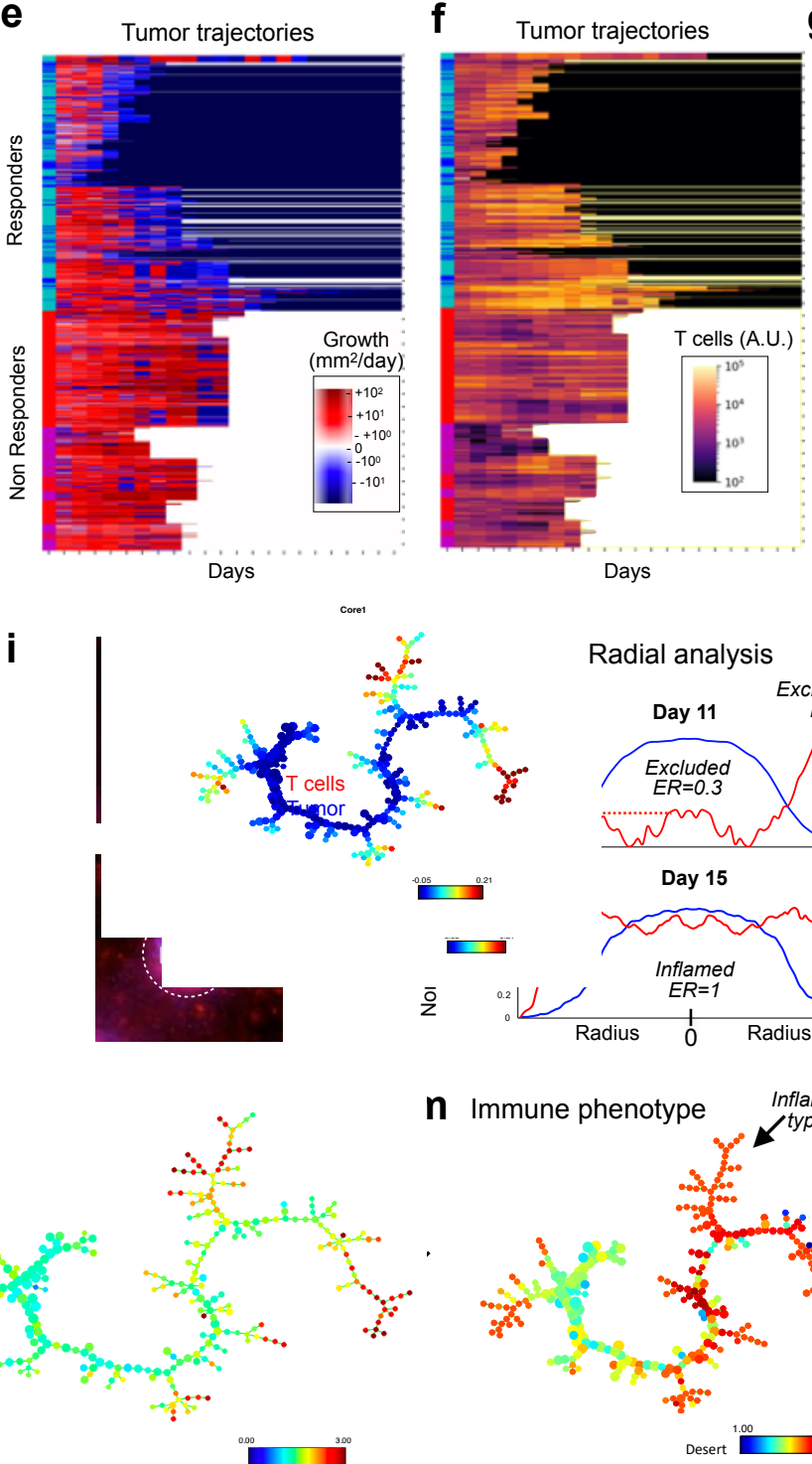

Days

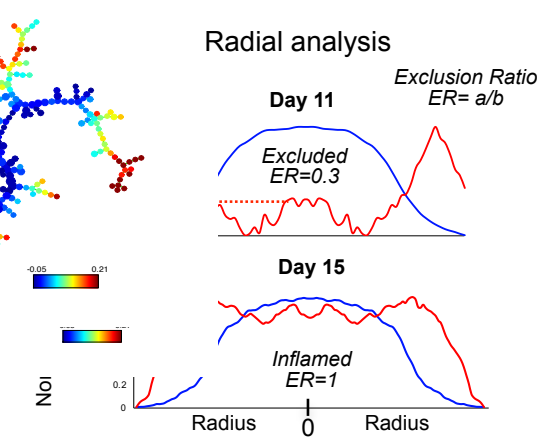

m Immune phenotype Inflamed

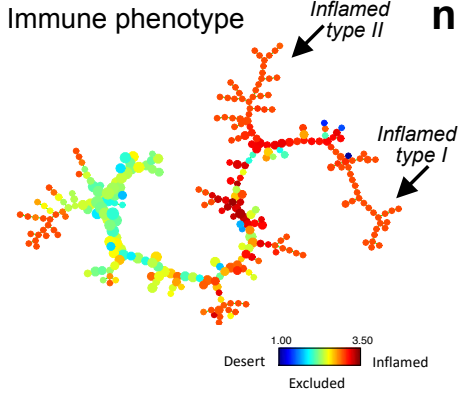

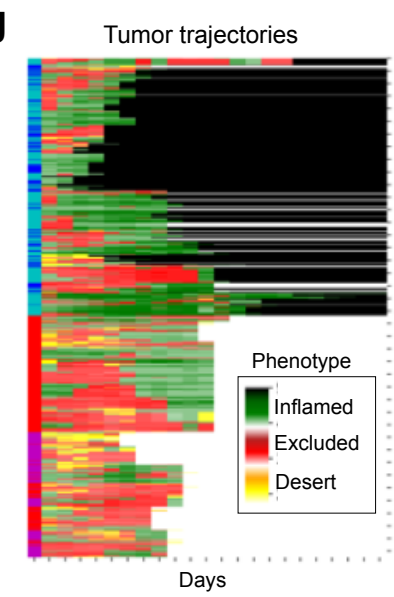

h

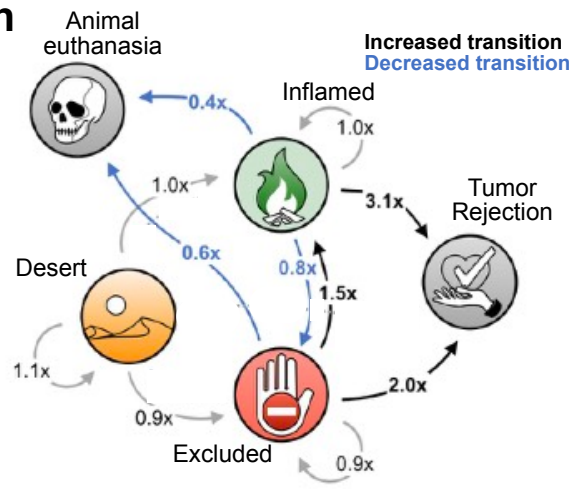

Fold differences in transition probabilities aTGF $\beta /$ aPD-L1 versus Isotype Control k

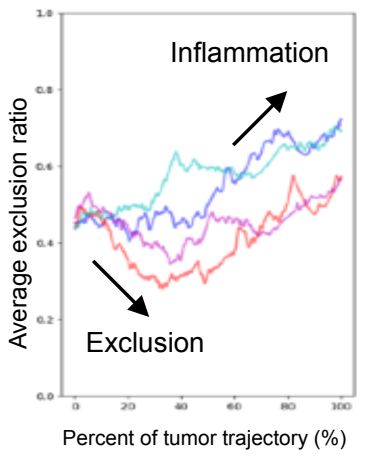

- Total T cell abundance

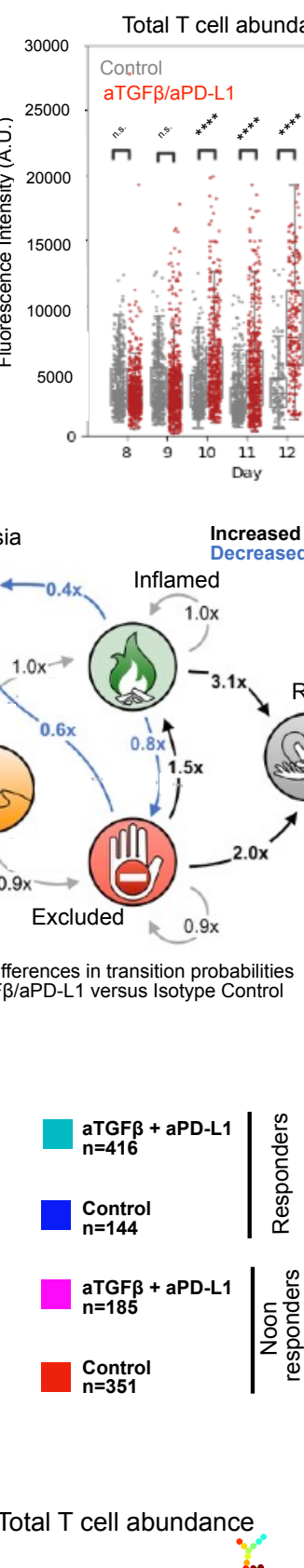

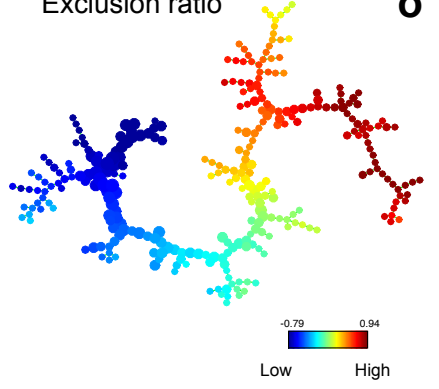

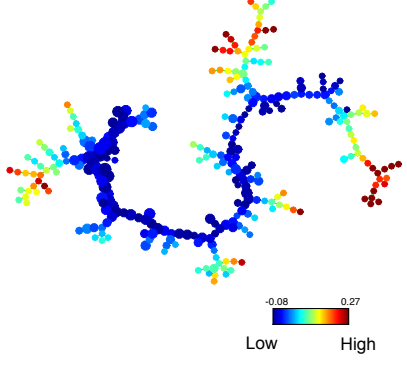

(

$9 \quad$ NSCLC Maxillofacial Melanoma $\underset{\text { panck Melanoma }}{\text { CD8 }}$

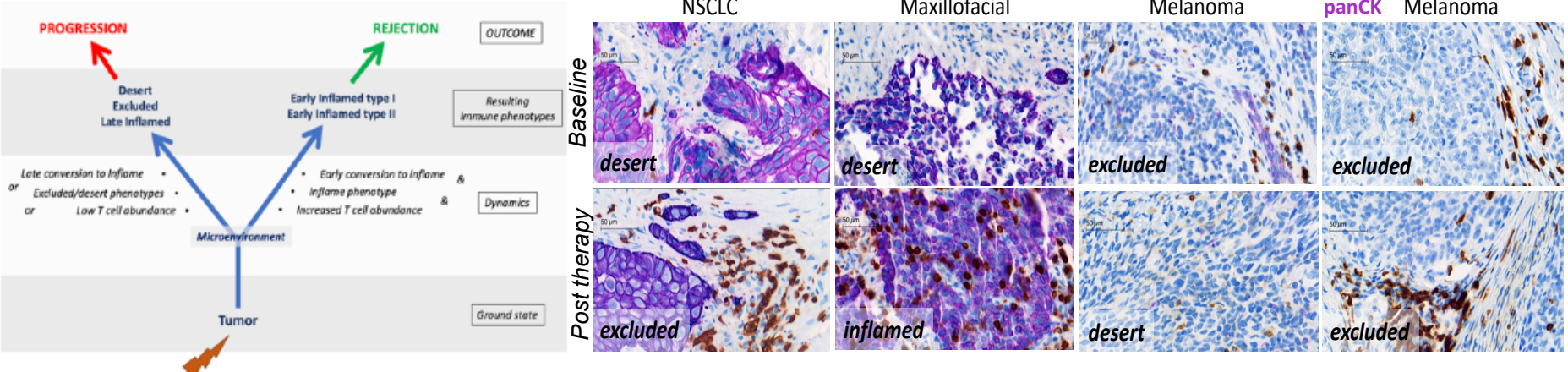


Fig. 4 | Early transition to an inflamed phenotype predicts response to Immunotherapy

a, I Kaplan Meier survival curves of individual tumors implanted in Rag2 KO animals reconstituted with tdTomato T cells and and treated at day 1 post-implantation with isotype control antibodies, anti-TGF- $\beta$, anti-PD-L1, or a combination of anti-PD-L1 with anti-TGF- $\beta$. $n \geqq 287$ tumors per group and 10-12 animals per group. Shaded area=95\% confidence interval, log-rank test p-value n.s. $>0.5,{ }^{* * * *}<0.0001$. b, Individual tumor growth kinetics $\left(\mathrm{mm}^{\wedge} 2\right)$ of control and combination anti-PD-L1 with anti-TGF- $\beta$ treated tumors. $n \geqq 287$ tumors per group and 10-12 animals per group. c, Maximum area achieved of Responders (C.R + P.R) and Non Responders (S.D+P.D) tumors for combination anti-PD-L1 with anti-TGF- $\beta$ versus isotype control treated animals as described in (b). d, Total T cell abundance over time (tdTomato MFI per tumor) for individual tumors for isotype control treated tumors (gray dots showed as reference) and combo anti-PD-L1/ anti-TGF- $\beta$ treated tumors (red dots). e-g, Hierarchical clustering of individual tumor immune trajectories for isotype control treated tumors and combo anti-PD-L1/anti-TGF- $\beta$ treated tumors. black=tumor resolved, white=mouse euthanized. Combo Responders (C.R. + P.R.) in cyan, combo Non Responders (S.D. + P.D.) in magenta. Control Responders (C.R. + P.R.) in blue, control Non Responders (S.D. + P.D.) in red. e, Tumor growth rate ( $\mathrm{mm} 2 /$ day) over time f, T cell abundance (TdTomato MFI per tumor over time) $\mathbf{g}$, Tumor Immune phenotype over time $\mathbf{h}$. Markov chain showing the fold difference between transition matrices for isotype control versus combination anti-PD-L1 with antiTGF- $\beta$ treated animals as described in (b), i, Epifluorescence images of the same individual tumor at day 11 and day 15 with overlaid of radial analysis. Tumor was selected from an isotype control treated animal from the experiment described in (a). Tumor=blue, $T$ cells=red. j, quantification of median radial fluorescence profile for individual tumor shown in (i) at day 11 (top) and day 15 (bottom) to distinguish T cell excluded (low T cell exclusion ratio) and inflamed phenotype (high T cell exclusion ratio) $\mathbf{k}$, average exclusion ratio over normalized tumor trajectory for Combo Responders (C.R. + P.R.) in cyan, combo Non Responders (S.D. + P.D.) in magenta. Control Responders (C.R. + P.R.) in blue, control Non Responders (S.D. + P.D.) in red. I, SPADE tree representing the layout of $11 \mathrm{~K}$ individual tumors across days 6-17 for all treatment groups, in the space of the four parameters (Total T cell abundance, T cell abundance in the tumor core; tumor exclusion ratio; Tumor size). SPADE trees were color-coded according to the response to therapy (panel I), Immune phenotype (panel $\mathbf{m}$ ) exclusion ratio (panel $\mathbf{n}$ ) and T cell abundance (panel o). Categorical variables such, e.g. phenotype or response, were converted to numerical variables that were further used to color the SPADE tree. Size of the dots is proportional to the number of tumors $\mathbf{p}$, graphical summary of Immune dynamics leading to outcome $q$, Histology of patient paired tumor biopsies at baseline and at progression after treatment with checkpoint blockades. CD8 staining in brown, pan-Cytokeratin staining in magenta. 